Pacific Northwest National Laboratory

Operated by Battelle for the

U.S. Department of Energy

\section{Hanford Site Environmental Surveillance Master Sampling Schedule for Calendar Year 2006}

L. E. Bisping

January 2006

Prepared for the U.S. Department of Energy under Contract DE-AC05-76RL01830 


\title{
DISCLAIMER
}

This report was prepared as an account of work sponsored by an agency of the United States Government. Reference herein to any specific commercial product, process, or service by trade name, trademark, manufacturer, or otherwise does not necessarily constitute or imply its endorsement, recommendation, or favoring by the United States Government or any agency thereof, or Battelle Memorial Institute. This report is a summary of major or significant activities occurring at the Hanford Site only, and is not a full disclosure of all details associated with Hanford-related activities, nor a substitute for legally required information subject to reporting requirements regarding releases, violations, etc.

\author{
PACIFIC NORTHWEST NATIONAL LABORATORY \\ operated by \\ BATTELLE \\ for the \\ UNITED STATES DEPARTMENT OF ENERGY \\ under Contract DE-AC05-76RL01830
}

Printed in the United States of America
May be available to DOE and DOE contractors from the
Office of Scientific and Technical Information,
P.O. Box 62, Oak Ridge, TN 37831-0062;
ph: (865) 576-8401
fax: (865) 576-5728
email: reports@adonis.osti.gov

\author{
May be available to the public from the National Technical Information Service, \\ U.S. Department of Commerce, 5285 Port Royal Rd., Springfield, VA 22161 \\ ph: (800) 553-6847 \\ fax: (703) 605-6900 \\ email: orders@ntis.fedworld.gov \\ online ordering: http://www.ntis.gov/ordering.htm
}

This document was printed on recycled paper. 


\title{
Hanford Site Environmental Surveillance Master Sampling Schedule for Calendar Year 2006
}

\author{
L. E. Bisping
}

January 2006

Prepared for the U.S. Department of Energy under Contract DE-AC05-76RL01830

Pacific Northwest National Laboratory

Richland, Washington 99352 


\section{Summary}

Environmental surveillance of the Hanford Site and surrounding areas is conducted by the Pacific Northwest National Laboratory (PNNL) ${ }^{(\mathrm{a})}$ for the U.S. Department of Energy (DOE). Sampling is conducted to evaluate levels of radioactive and nonradioactive pollutants in the Hanford environs, as required in DOE Order 450.1, Environmental Protection Program, and DOE Order 5400.5, Radiation Protection of the Public and the Environment. The sampling design is described in the Hanford Site Environmental Monitoring Plan (DOE/RL-91-50, U.S. Department of Energy, Richland Operations Office, Richland, Washington). The changing mission at Hanford from plutonium production to clean-up has resulted in decreases in radiological sampling scope over the past ten years. External radiation surveillance was discontinued at the end of CY2005.

This document contains the calendar year 2006 schedules for the routine and non-routine collection of samples for the Surface Environmental Surveillance Project (SESP) and Drinking Water Monitoring Project. Each section includes sampling locations, sample types, and analyses to be performed. In some cases, samples are scheduled on a rotating basis and may not be collected in 2006 in which case the anticipated year for collection is provided. In addition, a map showing approximate sampling locations is included for each media scheduled for collection in 2006.

\section{SESP Sampling}

The SESP is a multimedia environmental surveillance effort to measure the concentrations of radionuclides and chemicals in environmental media and assess the integrated effects of these materials on the environment and the public. Project staff collect samples of air, surface water, agricultural products, wildlife, and sediments. In addition, soil and natural vegetation samples are collected approximately every 5 years. The project also includes the capability to monitor particulate matter mass

concentrations in air. Analytical capabilities include the measurement of radionuclides at very low environmental concentrations and, in selected media, nonradiological chemicals including metals, anions, and volatile organic compounds.

(a) Pacific Northwest National Laboratory is operated by Battelle Memorial Institute for the U.S. Department of Energy under Contract DE-AC05-76RL01830. 


\section{Drinking Water Monitoring Project Sampling}

Fluor Hanford, Inc. is responsible for monitoring the quality of drinking water supplied by the DOE to its onsite facilities in accordance with federal and state regulations. PNNL conducts radiological monitoring of onsite drinking water for Fluor Hanford, Inc. concurrent with SESP activities to promote sampling efficiency and consistency, utilize expertise developed over the years, and reduce costs associated with management, sample collection procedure development, analytical contracting, data management, quality control, and reporting.

\section{Data Management}

The Hanford Environmental Information System (HEIS) database is used as a repository for data gathered during environmental surveillance activities at the Hanford Site. For ease in retrieving SESP or drinking water data from the HEIS database, the majority of the location names in this document are the location names used in the database.

\section{Schedule Changes}

This schedule is subject to modification during the year in response to changes in site operations, program requirements, and the nature of the observed results. Operational limitations such as weather, mechanical failures, sample availability, etc., may also impact scheduled sampling. Therefore, this document may not be an accurate record of samples collected during the year.

\section{Co-Samples}

Samples that are co-sampled and analyzed by both PNNL and the Washington State Department of Health (DOH) are indicated in the schedule, as are samples that are co-sampled and analyzed by both PNNL and the U.S. Food and Drug Administration (FDA).

\section{Additional Information}

Questions relating to the content of this document can be directed to T. M. Poston, Manager, Surface Environmental Surveillance Project, (509) 376-5678 or R.W. (Bill) Hanf, Manager, Drinking Water Monitoring Project, (509) 376-8264. 


\section{Contents}

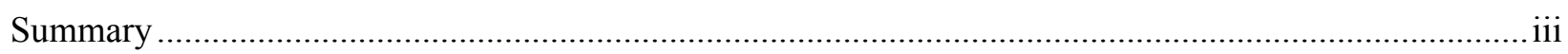

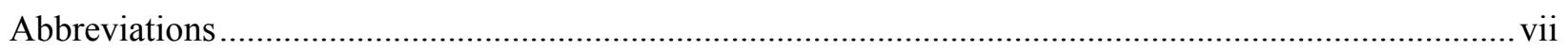

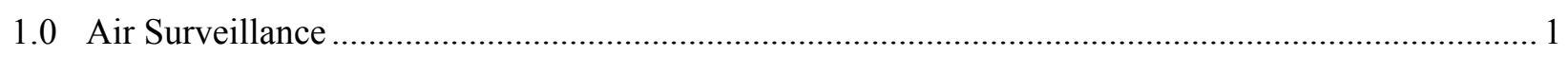

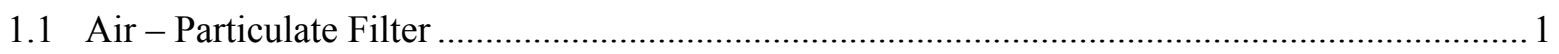

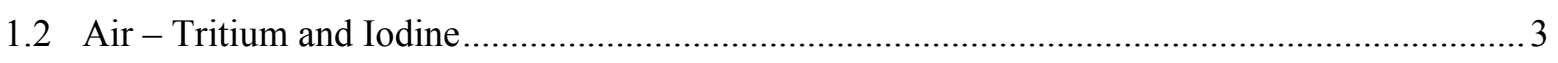

1.3 Air - Particulate Mass Concentration ................................................................................ 3

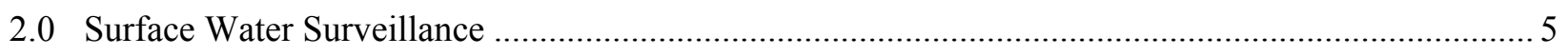

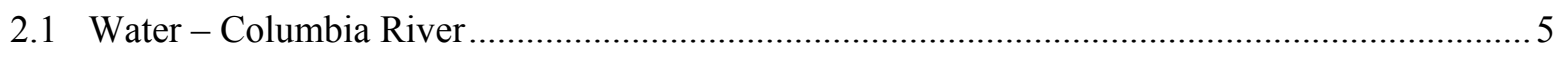

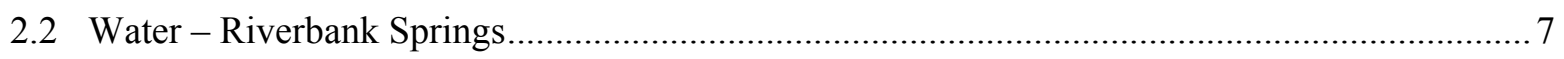

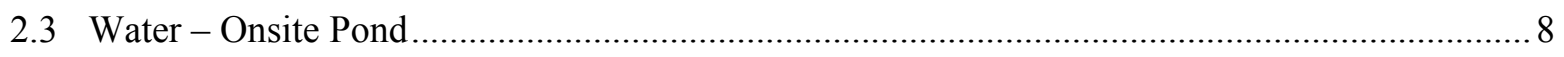

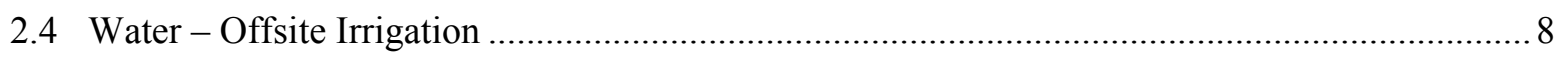

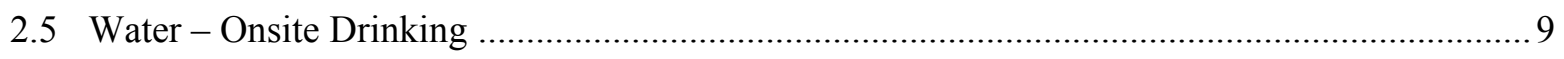

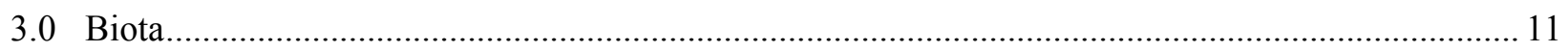

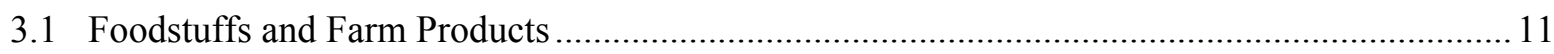

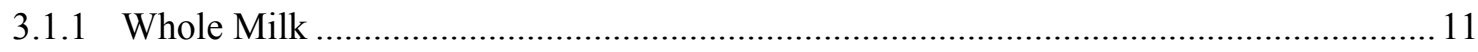

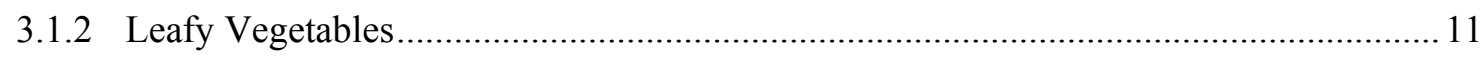

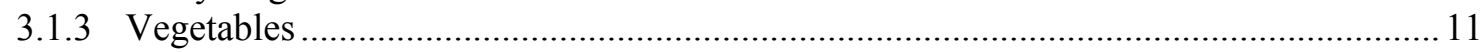

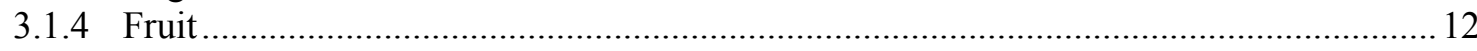

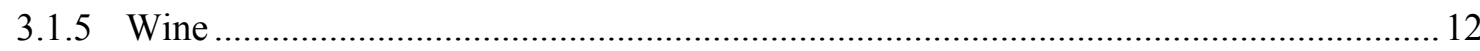

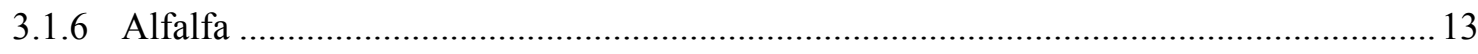

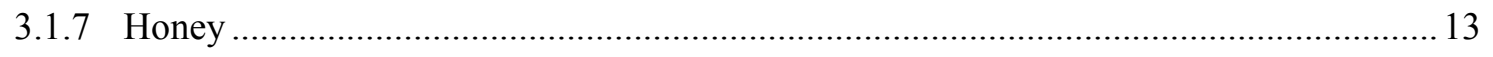

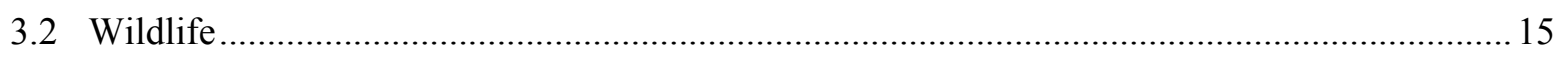

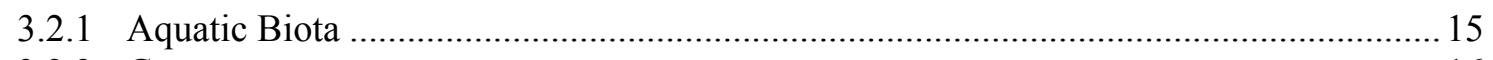

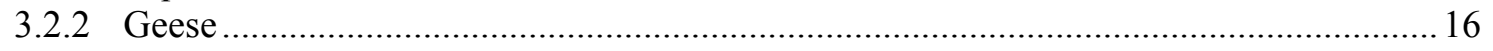

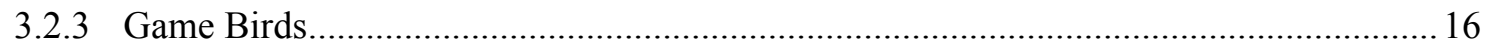

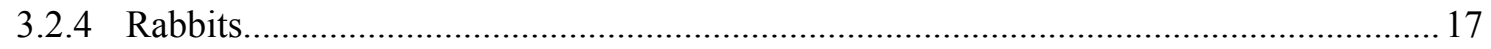

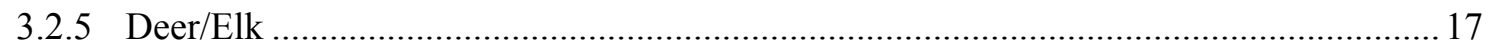

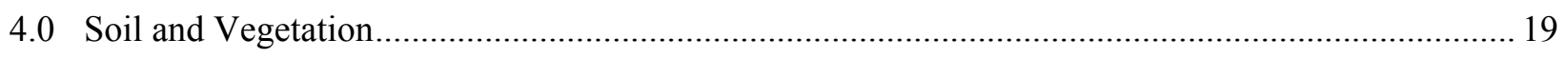

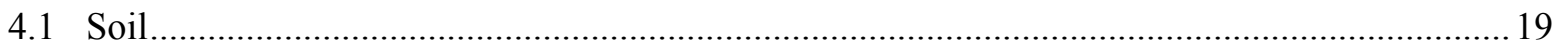

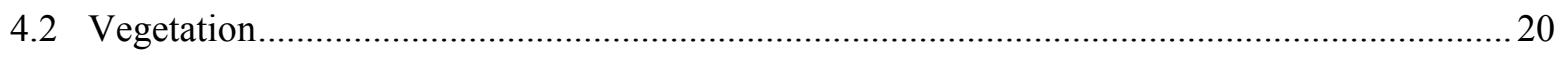

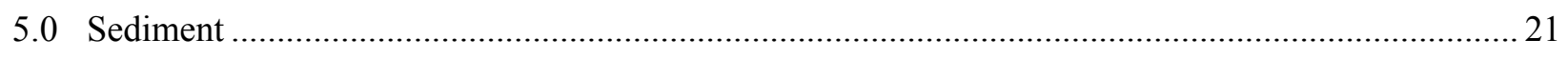

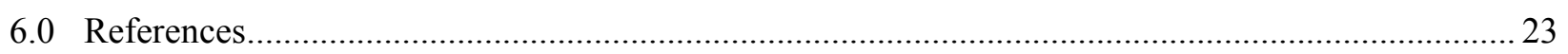




\section{Figures}

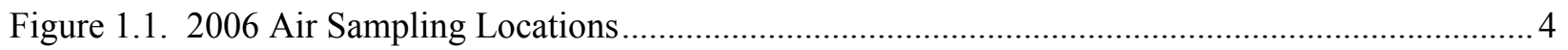

Figure 2.1. 2006 Surface Water and Drinking Water Sampling Locations .......................................... 10

Figure 3.1. 2006 Food and Farm Product Sampling Locations ............................................................. 14

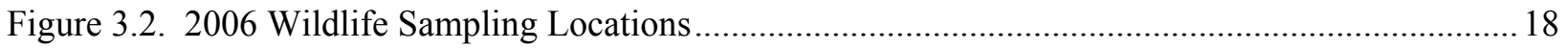

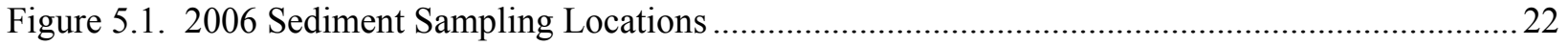




\section{Abbreviations}

\section{Frequency Symbols Used}

$\begin{array}{ll}\text { A } & \text { annually } \\ \text { BE } & \text { biennially (every 2 years) } \\ \text { BW } & \text { biweekly (every 2 weeks) } \\ \text { M } & \text { monthly } \\ \text { M Comp. } & \text { monthly composite } \\ \text { Q } & \text { quarterly } \\ \text { Q Comp. } & \text { quarterly composite } \\ \text { SA } & \text { semiannually (twice each year) } \\ \text { TE } & \text { triennially (every 3 years) }\end{array}$

\section{Analytical Symbols Used}

Generally, standard element, chemical, and isotope designations are used to indicate the analyses performed. Other analytical designations used are:

$\begin{array}{ll}\text { Alpha } & \text { gross alpha activity of sample } \\ \text { Anions } & \text { major anions-generally chloride, fluoride, nitrate, nitrite, sulfate } \\ \text { Beta } & \text { gross beta activity of sample } \\ \text { DDD } & \text { dichlorodiphenyl dichloroethane } \\ \text { DDE } & \text { dichlorodiphenyl dichloroethylene } \\ \text { DDT } & \text { dichlorodiphenyl trichloroethane } \\ \text { Gamma Scan } & \begin{array}{l}\text { analysis of photon energy spectrum for individual photon-emitting } \\ \text { radionuclides }\end{array} \\ \text { HTO } & \text { tritiated water }\left({ }^{3} \mathrm{H}^{1} \mathrm{H}^{16} \mathrm{O}\right) \\ \mathrm{Hg}-\mathrm{CVAA} & \text { mercury by cold vapor atomic absorbance spectrometry } \\ \mathrm{Hg}-\mathrm{CVAF} & \text { total mercury in water by cold vapor atomic fluorescence } \\ \text { ICP-MS } & \text { major metals by inductively coupled plasma mass spectrometry - samples } \\ & \text { unfiltered unless otherwise noted } \\ \text { Lo }{ }^{3} \mathrm{H} & \text { analytical procedure includes electrolytic enrichment } \\ \mathrm{PM} & \text { particulates having an aerodynamic diameter less than } 10 \text { and } \\ & \text { 2.5 micrometers respectively. Note that a PM } \mathrm{M}_{10} \text { sample includes } \mathrm{PM}_{2.5} \\ & \text { particulates } \\ \mathrm{Pu} & \text { isotopic plutonium }\left({ }^{238} \mathrm{Pu},{ }^{239 / 240} \mathrm{Pu}\right) \\ \mathrm{SEM} / \mathrm{AVS} & \text { simultaneously extracted metals/acid volatile sulfide } \\ \text { TOC } & \text { total organic carbon } \\ \mathrm{U} & \text { isotopic uranium }\left({ }^{234} \mathrm{U},{ }^{235} \mathrm{U},{ }^{238} \mathrm{U}\right) \\ \text { VOA } & \text { volatile organic compounds }\end{array}$




\subsection{Air Surveillance}

\subsection{Air - Particulate Filter}

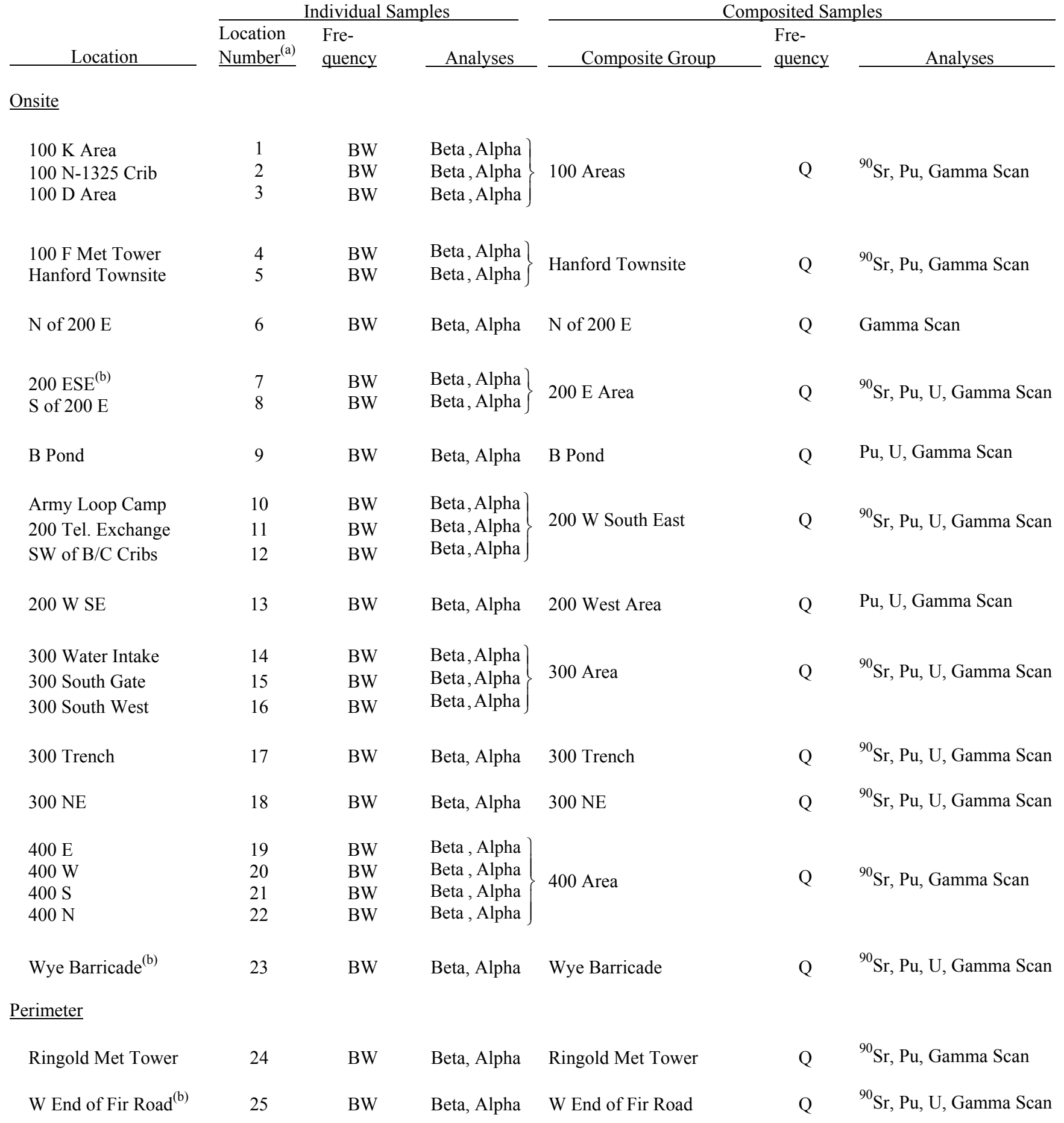




\subsection{Air - Particulate Filter (contd)}

\begin{tabular}{|c|c|c|c|c|c|c|}
\hline \multirow[b]{2}{*}{ Location } & \multicolumn{3}{|c|}{ Individual Samples } & \multicolumn{3}{|c|}{ Composited Samples } \\
\hline & $\begin{array}{l}\text { Location } \\
\text { Number }^{(a)}\end{array}$ & $\begin{array}{l}\text { Fre- } \\
\text { quency }\end{array}$ & Analyses & Composite Group & $\begin{array}{l}\text { Fre- } \\
\text { quency }\end{array}$ & Analyses \\
\hline Dogwood Met Tower & 26 & BW & Beta, Alpha & Dogwood Met Tower & Q & ${ }^{90} \mathrm{Sr}, \mathrm{U}$, Gamma Scan \\
\hline Battelle Complex $^{(b)}$ & 28 & $\mathrm{BW}$ & Beta, Alpha & Battelle Complex & Q & Gamma Scan \\
\hline $\begin{array}{l}\text { Horn Rapids Substa } \\
\text { Prosser Barricade } \\
\text { (b) }^{2}\end{array}$ & $\begin{array}{l}29 \\
30\end{array}$ & $\begin{array}{l}\text { BW } \\
\text { BW }\end{array}$ & $\left.\begin{array}{l}\text { Beta, Alpha } \\
\text { Beta, Alpha }\end{array}\right\}$ & Prosser Barricade & Q & ${ }^{90} \mathrm{Sr}, \mathrm{Pu}, \mathrm{U}$, Gamma Scan \\
\hline $\begin{array}{l}\text { Wahluke Slope } \\
\text { S End Vernita Bridge }\end{array}$ & $\begin{array}{l}33 \\
34\end{array}$ & $\begin{array}{l}\text { BW } \\
\text { BW }\end{array}$ & $\left.\begin{array}{l}\text { Beta, Alpha } \\
\text { Beta, Alpha }\end{array}\right\}$ & Wahluke Slope & Q & ${ }^{90} \mathrm{Sr}, \mathrm{Pu}$, Gamma Scan \\
\hline \multicolumn{7}{|l|}{ Community } \\
\hline Basin City School & 35 & BW & Beta, Alpha & Basin City School & Q & ${ }^{90} \mathrm{Sr}, \mathrm{Pu}, \mathrm{U}$, Gamma Scan \\
\hline Leslie Groves-Rchlnd & 36 & BW & Beta, Alpha & Leslie Groves-Rchlnd & Q & ${ }^{90} \mathrm{Sr}, \mathrm{Pu}, \mathrm{U}$, Gamma Scan \\
\hline $\begin{array}{l}\text { Pasco } \\
\text { Kennewick-Ely Street }\end{array}$ & $\begin{array}{l}37 \\
38\end{array}$ & $\begin{array}{l}\text { BW } \\
\text { BW }\end{array}$ & $\begin{array}{l}\text { Beta } \\
\text { Beta, Alpha }\end{array}$ & Tri Cities & Q & ${ }^{90} \mathrm{Sr}, \mathrm{Pu}$, Gamma Scan \\
\hline \multicolumn{7}{|l|}{$\underline{\text { Distant }}$} \\
\hline Yakima & 42 & $\mathrm{BW}$ & Beta, Alpha & Yakima & Q & ${ }^{90} \mathrm{Sr}, \mathrm{Pu}, \mathrm{U}, \mathrm{Gamma}$ Scan \\
\hline
\end{tabular}




\subsection{Air - Tritium and Iodine}

\begin{tabular}{|c|c|c|c|c|c|}
\hline Location & $\begin{array}{l}\text { Location } \\
\text { Number }^{(a)} \\
\end{array}$ & Frequency $^{(b)}$ & $\underline{\text { Analysis }}$ & $\underline{\text { Frequency }}$ & $\underline{\text { Analysis }^{(c)}}$ \\
\hline \multicolumn{6}{|l|}{$\underline{\text { Onsite }}$} \\
\hline $100 \mathrm{~K}$ Area & 1 & & & M & ${ }^{3} \mathrm{H}$ \\
\hline 100 N-1325 Crib & 2 & & & M & ${ }^{3} \mathrm{H}$ \\
\hline 200 ESE & 7 & Q Comp. & ${ }^{129} \mathrm{I}$ & $\mathrm{M}$ & ${ }^{3} \mathrm{H}$ \\
\hline 200 Tel. Exchange & 11 & & & M & ${ }^{3} \mathrm{H}$ \\
\hline 300 Water Intake ${ }^{(d)}$ & 14 & & & M & ${ }^{3} \mathrm{H}$ \\
\hline 300 South Gate ${ }^{(\mathrm{e})}$ & 15 & & & $\mathrm{M}$ & ${ }^{3} \mathrm{H}$ \\
\hline 300 South West & 16 & & & M & ${ }^{3} \mathrm{H}$ \\
\hline 300 Trench & 17 & & & M & ${ }^{3} \mathrm{H}$ \\
\hline $300 \mathrm{NE}$ & 18 & & & M & ${ }^{3} \mathrm{H}$ \\
\hline $400 \mathrm{E}$ & 19 & & & M & ${ }^{3} \mathrm{H}$ \\
\hline \multicolumn{6}{|l|}{$\underline{\text { Perimeter }}$} \\
\hline Ringold Met Tower & 24 & Q Comp. & ${ }^{129} \mathrm{I}$ & M & ${ }^{3} \mathrm{H}$ \\
\hline W End of Fir Road & 25 & & & M & ${ }^{3} \mathrm{H}$ \\
\hline Dogwood Met Tower & 26 & & & M & ${ }^{3} \mathrm{H}$ \\
\hline Byers Landing & 27 & Q Comp. & ${ }^{129} \mathrm{I}$ & M & ${ }^{3} \mathrm{H}$ \\
\hline Battelle Complex ${ }^{(\mathrm{d})}$ & 28 & & & M & ${ }^{3} \mathrm{H}$ \\
\hline Prosser Barricade ${ }^{(\mathrm{d})}$ & 30 & & & M & ${ }^{3} \mathrm{H}$ \\
\hline Wahluke Slope & 33 & & & M & ${ }^{3} \mathrm{H}$ \\
\hline \multicolumn{6}{|l|}{$\underline{\text { Community }}$} \\
\hline Basin City School & 35 & & & M & ${ }^{3} \mathrm{H}$ \\
\hline Leslie Groves-Rchlnd & 36 & & & M & ${ }^{3} \mathrm{H}$ \\
\hline \multicolumn{6}{|l|}{$\underline{\text { Distant }}$} \\
\hline Yakima & 42 & Q Comp. & ${ }^{129} \mathrm{I}$ & M & ${ }^{3} \mathrm{H}$ \\
\hline
\end{tabular}

(a) Refer to Figure 1.1, 2006 Air Sampling Locations.

(b) Samples are collected monthly and composited for quarterly analyses.

(c) As HTO.

(d) Washington State Department of Health air sampler also at this location.

(e) Two tritium samples are collected from this location.

\subsection{Air - Particulate Mass Concentration}

\begin{tabular}{|c|c|c|c|}
\hline Location & $\begin{array}{l}\text { Location } \\
\text { Number }^{(a)}\end{array}$ & $\underline{\text { Frequency }}$ & Analysis $^{(b)}$ \\
\hline Hanford Meteorological Station & 43 & Hourly & Mass Concentration \\
\hline
\end{tabular}

(a) Refer to Figure 1.1, 2006 Air Sampling Locations.

(b) Hourly average mass concentration data are collected at the Hanford Meteorological Station for two size fractions, $\mathrm{PM}_{10}$ and $\mathrm{PM}_{2.5}$. These data are not available in the HEIS database but can be obtained from the SESP manager. 


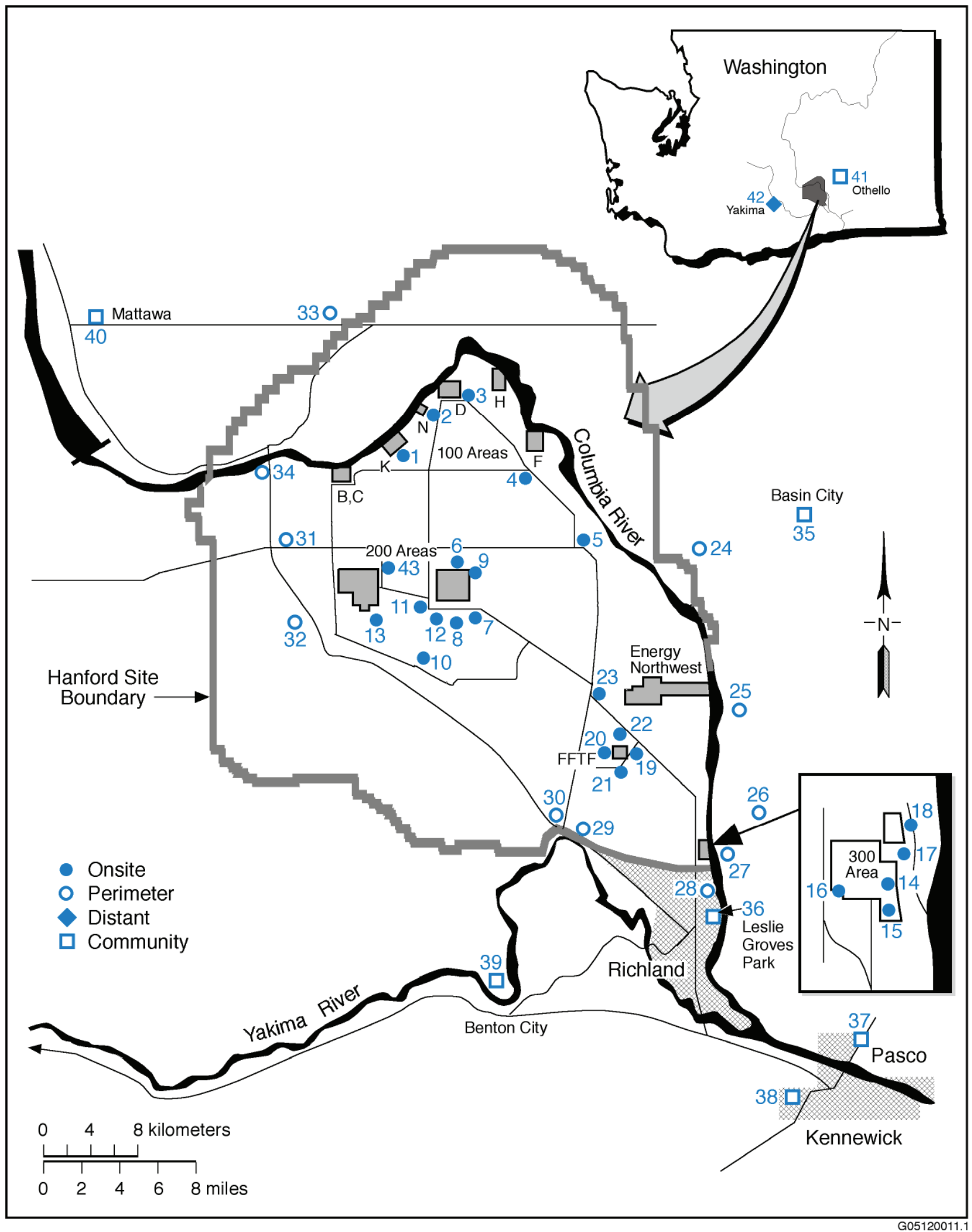

Figure 1.1. 2006 Air Sampling Locations 


\subsection{Surface Water Surveillance}

\subsection{Water - Columbia River}

\begin{tabular}{|c|c|c|c|}
\hline Location $^{(a)}$ & Sample Type & Frequency & Analyses/Co-Sample \\
\hline Priest Rapids-River & Cumulative & $\begin{array}{l}\text { M Comp. }{ }^{(b)} \\
\text { Q Comp. }\end{array}$ & $\begin{array}{l}\text { Alpha, Beta, Lo }{ }^{3} \mathrm{H},{ }^{90} \mathrm{Sr},{ }^{99} \mathrm{Tc}, \mathrm{U}, \mathrm{DOH}^{(\mathrm{c})} \\
{ }^{129} \mathrm{I}\end{array}$ \\
\hline & Particulate (filter) & $\begin{array}{l}\text { M Comp. } \\
\text { Q Comp. }^{(d)}\end{array}$ & $\begin{array}{l}\text { Gamma Scan } \\
\mathrm{Pu}\end{array}$ \\
\hline & Soluble (resin) & $\begin{array}{l}\text { M Comp. } \\
\text { Q Comp. }\end{array}$ & $\begin{array}{l}\text { Gamma San } \\
\mathrm{Pu}\end{array}$ \\
\hline Rich.Pmphs HRM 46.4 & Cumulative & $\begin{array}{l}\text { M Comp. }{ }^{(b)} \\
\text { Q Comp. }\end{array}$ & $\begin{array}{l}\text { Alpha, Beta, Lo }{ }^{3} \mathrm{H},{ }^{90} \mathrm{Sr},{ }^{99} \mathrm{Tc}, \mathrm{U} \\
{ }^{129} \mathrm{I}\end{array}$ \\
\hline & Particulate (filter) & $\begin{array}{l}\text { M Comp. } \\
\text { Q Comp. }^{(d)}\end{array}$ & $\begin{array}{l}\text { Gamma Scan } \\
\mathrm{Pu}\end{array}$ \\
\hline & Soluble (resin) & $\begin{array}{l}\text { M Comp. } \\
\text { Q Comp. }\end{array}$ & $\begin{array}{l}\text { Gamma Scan } \\
\mathrm{Pu}\end{array}$ \\
\hline & Grab & $3 / \mathrm{Yr}$ & USGS-NASQAN $^{(\mathrm{e})}$ \\
\hline Rich.Pmphs-1 HRM46.4 ${ }^{(\mathrm{f})}$ & Transect & $\begin{array}{l}\mathrm{Q} \\
\mathrm{A}\end{array}$ & $\begin{array}{l}\text { Lo }{ }^{3} \mathrm{H},{ }^{90} \mathrm{Sr}, \mathrm{U}, \text { Anions } \\
\text { ICP-MS, Hg-CVAF, ICP-MS Filtered, VOA }\end{array}$ \\
\hline Rich.Pmphs-2 HRM46.4 & Transect & $\mathrm{Q}$ & $\begin{array}{l}\text { Lo }{ }^{3} \mathrm{H},{ }^{90} \mathrm{Sr}, \mathrm{U}, \text { Anions } \\
\text { ICP-MS, Hg-CVAF, ICP-MS Filtered, VOA }\end{array}$ \\
\hline Rich.Pmphs-3 HRM46.4 & Transect & $\begin{array}{l}\text { Q } \\
\mathrm{A}\end{array}$ & $\begin{array}{l}\text { Lo }{ }^{3} \mathrm{H},{ }^{90} \mathrm{Sr}, \mathrm{U}, \text { Anions } \\
\text { ICP-MS, Hg-CVAF, ICP-MS Filtered, VOA }\end{array}$ \\
\hline Rich.Pmphs-5 HRM46.4 & Transect & $\begin{array}{l}\mathrm{Q} \\
\mathrm{A}\end{array}$ & $\begin{array}{l}\text { Lo }{ }^{3} \mathrm{H},{ }^{90} \mathrm{Sr}, \mathrm{U}, \text { Anions } \\
\text { ICP-MS, Hg-CVAF, ICP-MS Filtered, VOA }\end{array}$ \\
\hline Rich.Pmphs-7 HRM46.4 & Transect & $\begin{array}{l}\mathrm{Q} \\
\mathrm{A}\end{array}$ & $\begin{array}{l}\text { Lo }{ }^{3} \mathrm{H},{ }^{90} \mathrm{Sr}, \mathrm{U}, \text { Anions } \\
\text { ICP-MS, Hg-CVAF, ICP-MS Filtered, VOA }\end{array}$ \\
\hline Rich.Pmphs-10 HRM46.4 & Transect & Q & $\begin{array}{l}\text { Lo }{ }^{3} \mathrm{H},{ }^{90} \mathrm{Sr}, \mathrm{U}, \text { Anions } \\
\text { ICP-MS, Hg-CVAF, ICP-MS Filtered, VOA }\end{array}$ \\
\hline Rich.Pmphs HRM 43.5 & Transect & Q & $\begin{array}{l}\text { Lo }{ }^{3} \mathrm{H},{ }^{90} \mathrm{Sr}, \mathrm{U}, \text { Anions } \\
\text { ICP-MS, Hg-CVAF, ICP-MS Filtered, DOH }{ }^{(\mathrm{g})}\end{array}$ \\
\hline Rich.Pmphs HRM 43.9 & Transect & $\begin{array}{l}\text { Q } \\
\mathrm{A}\end{array}$ & $\begin{array}{l}\text { Lo }{ }^{3} \mathrm{H},{ }^{90} \mathrm{Sr}, \mathrm{U}, \text { Anions } \\
\text { ICP-MS, Hg-CVAF, ICP-MS Filtered, DOH }{ }^{(\mathrm{g})}\end{array}$ \\
\hline Rich.Pmphs HRM 45.0 & Transect & $\begin{array}{l}\mathrm{Q} \\
\mathrm{A}\end{array}$ & $\begin{array}{l}\text { Lo }{ }^{3} \mathrm{H},{ }^{90} \mathrm{Sr}, \mathrm{U} \text {, Anions } \\
\text { ICP-MS, Hg-CVAF, ICP-MS Filtered, DOH }{ }^{(\mathrm{g})}\end{array}$ \\
\hline Rich.Pmphs HRM 45.8 & Transect & $\begin{array}{l}\mathrm{Q} \\
\mathrm{A}\end{array}$ & $\begin{array}{l}\text { Lo }{ }^{3} \mathrm{H},{ }^{90} \mathrm{Sr}, \mathrm{U}, \text { Anions } \\
\text { ICP-MS, Hg-CVAF, ICP-MS Filtered, DOH }{ }^{(\mathrm{g})}\end{array}$ \\
\hline Vernita & Grab & $3 / \mathrm{Yr}$ & USGS-NASQAN $^{(\mathrm{e})}$ \\
\hline Vernita-1 HRM 0.3 & Transect & $\begin{array}{l}\mathrm{Q} \\
\mathrm{A}\end{array}$ & $\begin{array}{l}\text { Lo }{ }^{3} \mathrm{H},{ }^{90} \mathrm{Sr}, \mathrm{U}, \text { Anions } \\
\text { ICP-MS, Hg-CVAF, ICP-MS Filtered, VOA }\end{array}$ \\
\hline Vernita-2 HRM 0.3 & Transect & $\begin{array}{l}\mathrm{Q} \\
\mathrm{A}\end{array}$ & $\begin{array}{l}\text { Lo }{ }^{3} \mathrm{H},{ }^{90} \mathrm{Sr}, \mathrm{U}, \text { Anions } \\
\text { ICP-MS, Hg-CVAF, ICP-MS Filtered, VOA }\end{array}$ \\
\hline
\end{tabular}




\subsection{Water - Columbia River (contd)}

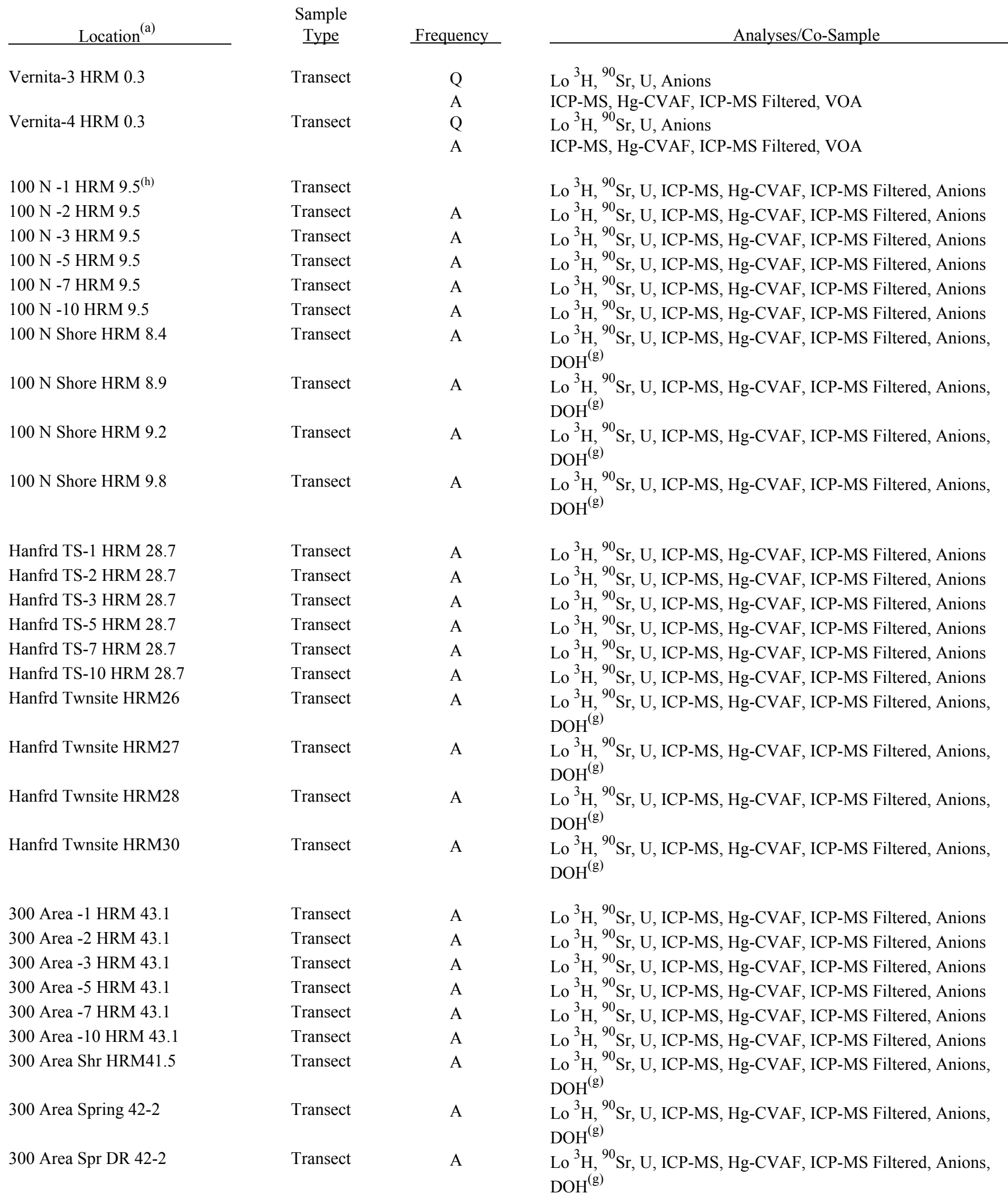




\subsection{Water - Columbia River (contd)}

\begin{tabular}{|c|c|c|c|}
\hline Location $^{(a)}$ & $\begin{array}{l}\text { Sample } \\
\text { Type }\end{array}$ & Frequency & Analyses/Co-Sample \\
\hline 300 Area Shr HRM42.9 & Transect & A & $\begin{array}{l}\text { Lo }{ }^{3} \mathrm{H},{ }^{90} \mathrm{Sr}, \mathrm{U}, \mathrm{ICP}-\mathrm{MS}, \mathrm{Hg}-\mathrm{CVAF}, \mathrm{ICP}-\mathrm{MS} \text { Filtered, Anions, } \\
\mathrm{DOH}^{(\mathrm{g})}\end{array}$ \\
\hline 300 Area Outfl13 & Grab & $\begin{array}{l}\mathrm{Q} \\
\mathrm{A}\end{array}$ & $\begin{array}{l}\text { Lo }{ }^{3} \mathrm{H},{ }^{90} \mathrm{Sr}, \mathrm{U}, \text { Anions } \\
\text { ICP-MS, Hg-CVAF, ICP-MS Filtered }\end{array}$ \\
\hline
\end{tabular}

(a) Refer to Figure 2.1, 2006 Surface Water and Drinking Water Sampling Locations. Hanford River Markers (HRM) are a series of signposts along the Hanford shoreline of the Columbia River that are roughly 1.6 kilometer $(1$ mile) apart. The Vernita Bridge is HRM \#0 and Ferry Street in Richland is HRM \#46. Samples collected between HRMs are assigned a decimal.

(b) Cumulative sample is collected weekly and composited for analysis.

(c) Co-sample provided to the Washington State Department of Health (January and June only).

(d) Sample is collected biweekly and composited for analysis.

(e) Analyses are performed by the United States Geological Survey (USGS) in conjunction with the National Stream Quality Accounting Network (NASQAN) Program, and include: conductance, $\mathrm{pH}$, temperature, turbidity, dissolved oxygen, hardness, Ca, Mg, alkalinity, carbonates, sulfate, $\mathrm{Cl}$, F, solids, $\mathrm{NH}_{4}-\mathrm{N}, \mathrm{NO}_{3}+\mathrm{NO}_{2}, \mathrm{~N}-\mathrm{Kjeldahl}, \mathrm{P}, \mathrm{Cr}, \mathrm{Fe}$, dissolved organic carbon.

(f) Quality assurance sample submitted for analyses twice per year.

(g) One co-sample provided to the Washington State Department of Health.

(h) Quality assurance sample submitted for analyses once per year.

\subsection{Water - Riverbank Springs}

\begin{tabular}{|c|c|c|}
\hline Location $^{(a)}$ & $\underline{H R M}^{(b)}$ & $\begin{array}{l}\text { Sample } \\
\text { Type }\end{array}$ \\
\hline 100-B Spring 38-3 & 3.8 & Grab \\
\hline 100-B Spring 39-2 & 3.9 & Grab \\
\hline 100-K Spring 63-1 & 6.3 & Grab \\
\hline 100-K Spring 77-1 & 7.6 & Grab \\
\hline $\begin{array}{c}\text { 100-N Spring Near } \\
199 N-46\end{array}$ & 8.9 & Grab \\
\hline $100-N$ Spring 8-13 & 9.3 & Grab \\
\hline 100-D Spring 102-1 & 10.2 & Grab \\
\hline 100-D Spring $110-1$ & 11.0 & Grab \\
\hline 100-H Spring 145-1 & 14.4 & Grab \\
\hline 100-H Spring 152-2 & 15.3 & Grab \\
\hline 100-F Spring 207-1 & 21.3 & Grab \\
\hline
\end{tabular}

\section{Analyses/Co-Sample}

Alpha, Beta, ${ }^{3} \mathrm{H},{ }^{90} \mathrm{Sr},{ }^{99} \mathrm{Tc}$, Gamma Scan, ICP-MS, Hg-CVAF, ICP-MS Filtered, Anions, VOA

Alpha, Beta, ${ }^{3} \mathrm{H},{ }^{90} \mathrm{Sr},{ }^{99} \mathrm{Tc}$, Gamma Scan, ICP-MS, Hg-CVAF, ICP-MS Filtered, Anions, VOA

Alpha, Beta, ${ }^{3} \mathrm{H},{ }^{90} \mathrm{Sr}$, Gamma Scan, ICP-MS, Hg-CVAF, ICP-MS Filtered, Anions, VOA, $\mathrm{DOH}^{(\mathrm{c})}$

Alpha, Beta, ${ }^{3} \mathrm{H},{ }^{90} \mathrm{Sr}$, Gamma Scan, ICP-MS, Hg-CVAF, ICP-MS Filtered, Anions, $\mathrm{VOA}, \mathrm{DOH}^{(\mathrm{c})}$

Alpha, Beta, ${ }^{3} \mathrm{H},{ }^{90} \mathrm{Sr}$, U, Gamma Scan, ICP-MS, Hg-CVAF, ICP-MS Filtered, Anions

Alpha, Beta, ${ }^{3} \mathrm{H},{ }^{90} \mathrm{Sr}$, Gamma Scan, ICP-MS, Hg-CVAF, ICP-MS Filtered, Anions, $\mathrm{DOH}^{(\mathrm{c})}$

Alpha, Beta, ${ }^{3} \mathrm{H},{ }^{90} \mathrm{Sr}$, Gamma Scan, ICP-MS, Hg-CVAF, ICP-MS Filtered, Anions

Alpha, Beta, ${ }^{3} \mathrm{H},{ }^{90} \mathrm{Sr}$, Gamma Scan, ICP-MS, Hg-CVAF, ICP-MS Filtered, Anions

Alpha, Beta, ${ }^{3} \mathrm{H},{ }^{90} \mathrm{Sr},{ }^{99} \mathrm{Tc}, \mathrm{U}$, Gamma Scan, ICP-MS, Hg-CVAF, ICP-MS Filtered, Anions

Alpha, Beta, ${ }^{3} \mathrm{H},{ }^{90} \mathrm{Sr},{ }^{99} \mathrm{Tc}, \mathrm{U}$, Gamma Scan, ICP-MS, Hg-CVAF, ICP-MS Filtered, Anions

Alpha, Beta, ${ }^{3} \mathrm{H},{ }^{90} \mathrm{Sr}, \mathrm{U}$, Gamma Scan, ICP-MS, Hg-CVAF, ICP-MS Filtered, Anions, VOA 


\subsection{Water - Riverbank Springs (contd)}

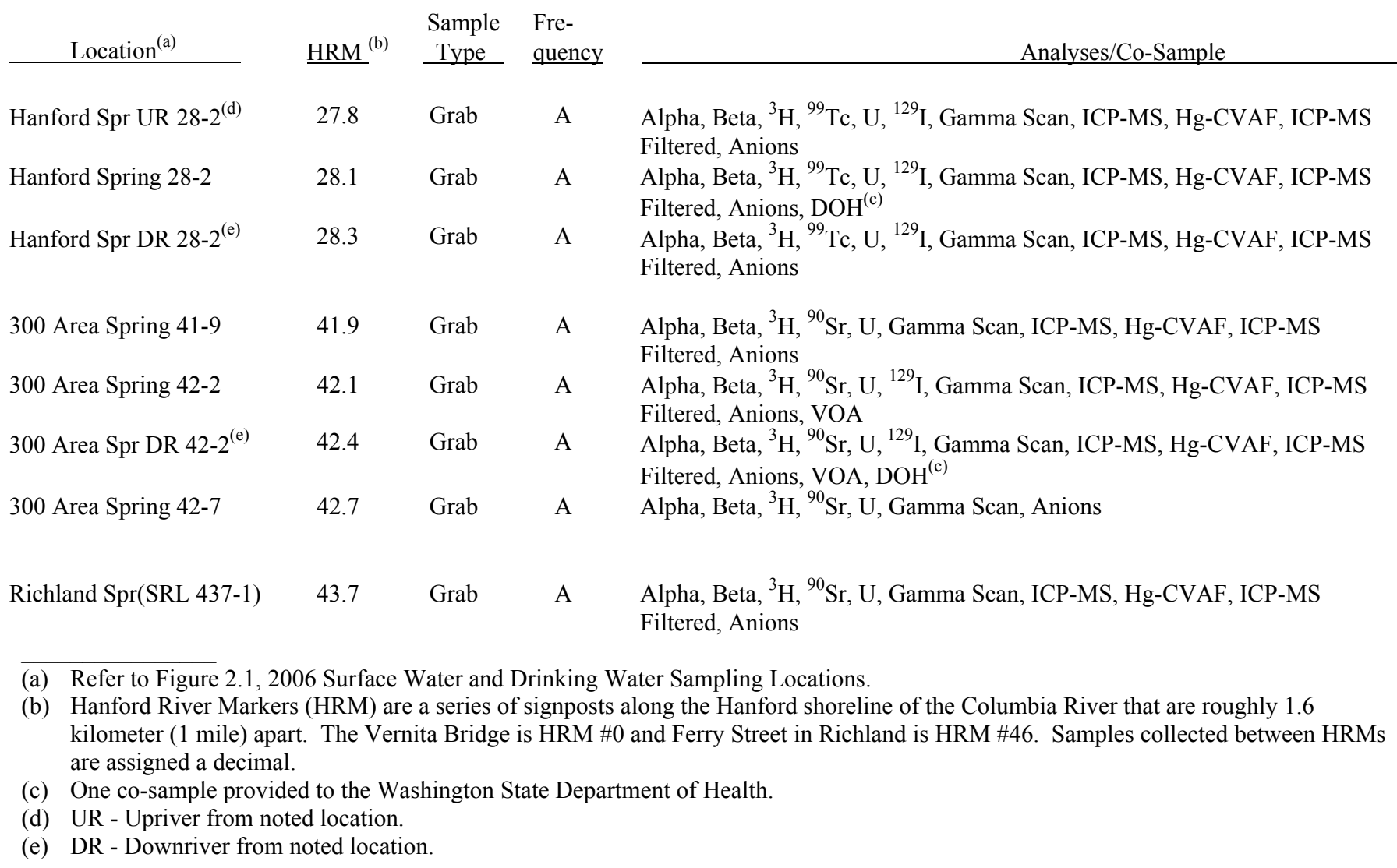

\subsection{Water - Onsite Pond}

\begin{tabular}{|c|c|c|c|}
\hline Location $^{(a)}$ & Sample Type & Frequency & Analyses \\
\hline West Lake & Grab & Q & ${ }^{3} \mathrm{H}$ \\
\hline FFTF Pond ${ }^{(b)}$ & Grab & Q & Alpha, Beta, ${ }^{3} \mathrm{H}$, Gamma Scan \\
\hline
\end{tabular}

(a) Refer to Figure 2.1, 2006 Surface Water and Drinking Water Sampling Locations.

(b) Quality assurance sample submitted for analyses once per year.

\subsection{Water - Offsite Irrigation}

\begin{tabular}{|c|c|c|c|}
\hline Location $^{(a)}$ & Sample Type & Frequency & Analyses/Co-Sample \\
\hline Riverview Canal & Grab & 3 (May-Sept) & Alpha, Beta, Lo ${ }^{3} \mathrm{H},{ }^{90} \mathrm{Sr}, \mathrm{U}$, Gamma Scan, DOH ${ }^{(\mathrm{b})}$ \\
\hline Horn Rapids Area & Grab & 3 (May-Sept) & Alpha, Beta, Lo ${ }^{3} \mathrm{H},{ }^{90} \mathrm{Sr}, \mathrm{U}$, Gamma Scan, DOH ${ }^{(\mathrm{b})}$ \\
\hline
\end{tabular}

(a) Refer to Figure 2.1, 2006 Surface Water and Drinking Water Sampling Locations.

(b) One co-sample provided to the Washington State Department of Health. 


\subsection{Water - Onsite Drinking}

\begin{tabular}{|c|c|c|c|c|c|c|c|}
\hline \multirow[b]{2}{*}{ Location $^{(\mathrm{a})}$} & \multirow[b]{2}{*}{$\begin{array}{c}\text { Sample } \\
\text { Type }\end{array}$} & \multicolumn{3}{|c|}{ Individual Samples } & \multicolumn{3}{|c|}{ Composited Samples } \\
\hline & & $\begin{array}{l}\text { Fre- } \\
\text { quency }\end{array}$ & Analyses/Co-Sam & & $\underline{\text { Composite Group }}$ & $\begin{array}{l}\text { Fre- } \\
\text { quency }\end{array}$ & $\underline{\text { Analyses }}$ \\
\hline $100 \mathrm{~N}$ Area & Grab & $M^{(b)}$ & & \} & $100 \mathrm{~N}$ Area & Q & Beta \\
\hline $100 \mathrm{~N}$ Area & Grab & $Q^{(c)}$ & Alpha, ${ }^{226} \mathrm{Ra},{ }^{228} \mathrm{Ra},{ }^{131} \mathrm{I}$ & \} & $100 \mathrm{~N}$ Area & A & ${ }^{90} \mathrm{Sr},{ }^{3} \mathrm{H}$ \\
\hline 200 W Area & Grab & $\mathrm{M}^{(\mathrm{b})}$ & & \} & $200 \mathrm{~W}$ Area & Q & Beta \\
\hline 200 W Area & Grab & $Q^{(c)}$ & Alpha, ${ }^{226} \mathrm{Ra},{ }^{228} \mathrm{Ra},{ }^{131} \mathrm{I}$ & \} & $200 \mathrm{~W}$ Area & A & ${ }^{90} \mathrm{Sr},{ }^{3} \mathrm{H}$ \\
\hline $100 \mathrm{~K}$ Area & Grab & $M^{(b)}$ & & \} & $100 \mathrm{~K}$ Area & Q & Beta \\
\hline $100 \mathrm{~K}$ Area & Grab & $Q^{(c)}$ & Alpha, ${ }^{226} \mathrm{Ra},{ }^{228} \mathrm{Ra},{ }^{131} \mathrm{I}$ & \} & $100 \mathrm{~K}$ Area & A & ${ }^{90} \mathrm{Sr},{ }^{3} \mathrm{H}$ \\
\hline 400 Area & Grab & $M^{(b)}$ & & \} & 400 Area & Q & Beta \\
\hline 400 Area & Grab & $Q^{(c)}$ & Alpha, ${ }^{226} \mathrm{Ra},{ }^{228} \mathrm{Ra},{ }^{131} \mathrm{I}$, & $\left.\mathrm{H}, \mathrm{DOH}^{(\mathrm{d})}\right\}$ & 400 Area & A & ${ }^{90} \mathrm{Sr}$ \\
\hline
\end{tabular}

(a) Refer to Figure 2.1, 2006 Surface Water and Drinking Water Sampling Locations.

(b) Sample is collected monthly and composited for quarterly analysis.

(c) Sample is collected and analyzed quarterly and then composited for annual analysis.

(d) One co-sample provided to the Washington State Department of Health for ${ }^{3} \mathrm{H}$ (January only). 


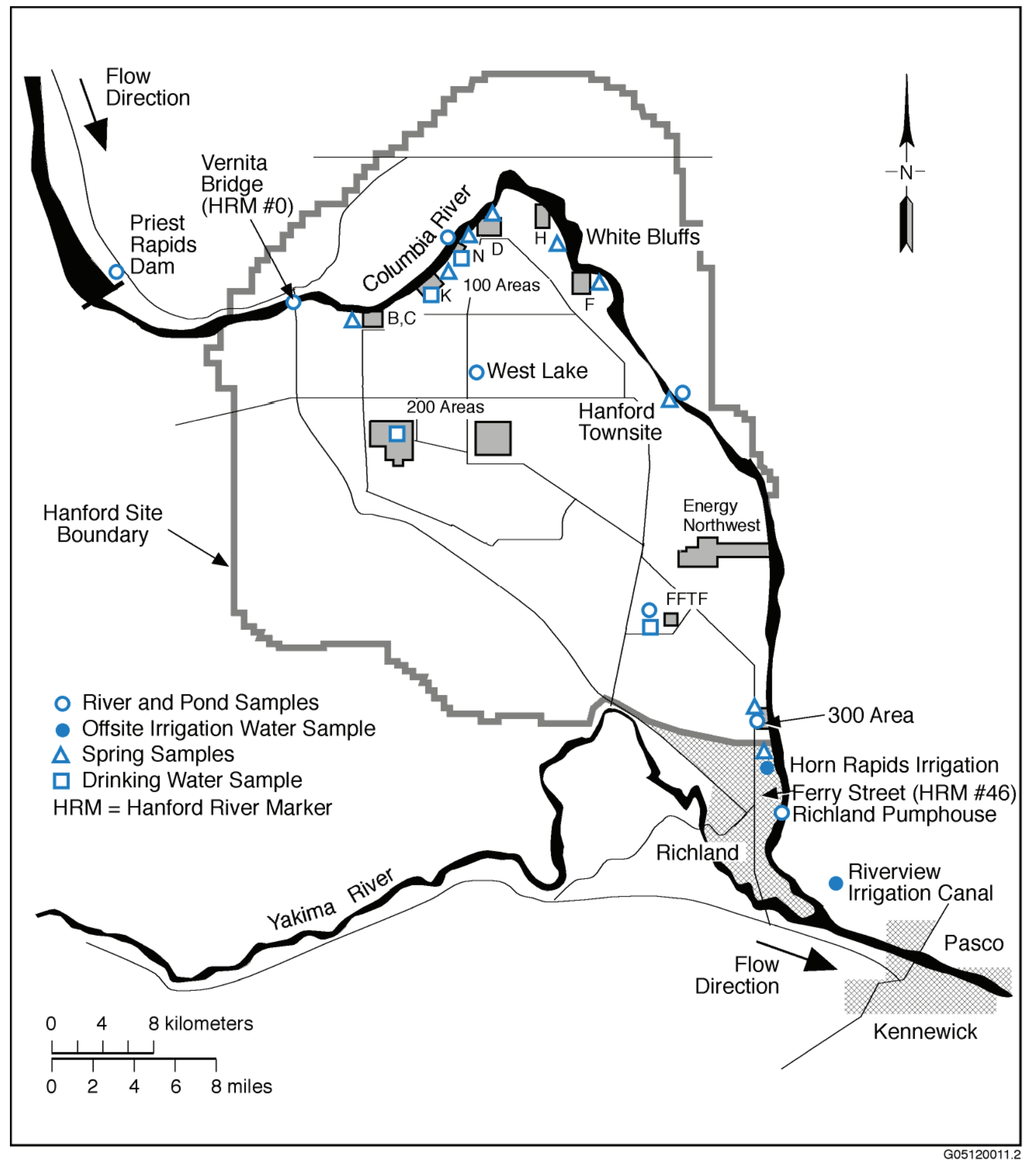

Figure 2.1. 2006 Surface Water and Drinking Water Sampling Locations 


\subsection{Biota}

\subsection{Foodstuffs and Farm Products}

\subsubsection{Whole Milk}

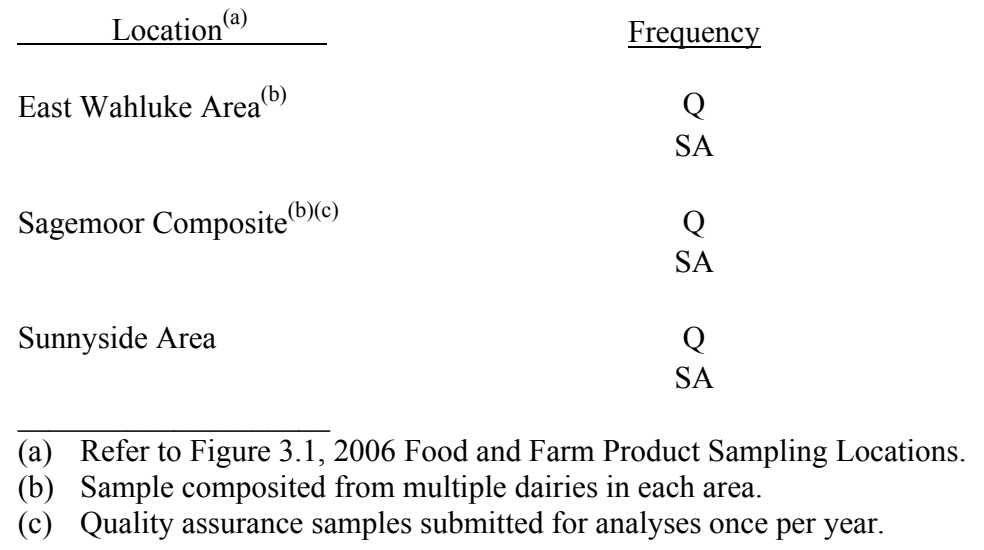

\subsubsection{Leafy Vegetables}

\section{Location $^{(a)(b)}$}

Riverview Area

Sunnyside Area

East Wahluke Area

Sagemoor Area

$$
\text { Frequency }^{(\mathrm{c})}
$$

\section{A}

A

BE (2006)

BE (2007)
Analyses

Lo ${ }^{3} \mathrm{H},{ }^{90} \mathrm{Sr}$, Gamma Scan

${ }^{129} \mathrm{I}$

Lo ${ }^{3} \mathrm{H},{ }^{90} \mathrm{Sr}$, Gamma Scan

${ }^{129} \mathrm{I}$

Lo ${ }^{3} \mathrm{H},{ }^{90} \mathrm{Sr}$, Gamma Scan

(a) Refer to Figure 3.1, 2006 Food and Farm Product Sampling Locations.

(b) Two samples collected for PNNL within each area, one sample analyzed and one archived.

(c) Samples are collected in 2006 according to their specified frequency unless otherwise noted.

(d) Two co-samples sent to U.S. Food and Drug Administration.

(e) One co-sample provided to the Washington State Department of Health.

\subsubsection{Vegetables}

\section{Location $^{(a)(b)}$}

Riverview Area

Sunnyside Area

East Wahluke Area

Harrah/Wapato Area

Sagemoor Area

Horn Rapids Area

\section{Sample Type}

Potatoes

Tomatoes

Asparagus

Potatoes

Asparagus

Potatoes

Tomatoes

Asparagus

Potatoes

Potatoes $\underline{\text { Frequency }}^{(\mathrm{c})}$

A

A

A

A

A

A

A

A

TE (2006)

TE (2008)
Analyses/Co-Sample

${ }^{90} \mathrm{Sr}$, Gamma Scan, FDA ${ }^{(\mathrm{d})}$

${ }^{90} \mathrm{Sr}$, Gamma Scan, FDA ${ }^{(\mathrm{d})}$

${ }^{90} \mathrm{Sr}$, Gamma Scan, $\mathrm{DOH}^{(\mathrm{e})}$

${ }^{90} \mathrm{Sr}$, Gamma Scan, DOH ${ }^{(\mathrm{e})}$

(a) Refer to Figure 3.1, 2006 Food and Farm Product Sampling Locations.

(b) Two samples collected for PNNL within each area, one sample analyzed and one archived.

(c) Samples are collected in 2006 according to their specified frequency unless otherwise noted.

(d) Two co-samples sent to U.S. Food and Drug Administration.

(e) One co-sample provided to the Washington State Department of Health. 


\subsubsection{Fruit}

\begin{tabular}{|c|c|c|c|c|}
\hline Location $^{(\mathrm{a})(\mathrm{b})}$ & Sample Type & Frequency $^{(\mathrm{c})}$ & $\begin{array}{c}\text { Collection } \\
\text { Period } \\
\end{array}$ & Analyses/Co-Sample \\
\hline Sagemoor Area & $\begin{array}{l}\text { Apples } \\
\text { Concord Grapes }^{(f)} \\
\text { Cherries }\end{array}$ & $\begin{array}{l}\text { TE (2006) } \\
\text { TE (2007) } \\
\text { TE (2008) }\end{array}$ & $\begin{array}{l}\text { September } \\
\text { September } \\
\text { June }\end{array}$ & $\begin{array}{l}{ }^{90} \mathrm{Sr}, \text { Gamma Scan, } \mathrm{DOH},{ }^{(\mathrm{d})} \mathrm{FDA}^{(\mathrm{e})} \\
{ }^{90} \mathrm{Sr}, \text { Gamma Scan, } \mathrm{DOH}^{(\mathrm{d})} \\
{ }^{90} \mathrm{Sr}, \text { Gamma Scan, } \mathrm{DOH}^{(\mathrm{d})}{ }^{\mathrm{FDA}}{ }^{(\mathrm{e})}\end{array}$ \\
\hline Sunnyside Area & $\begin{array}{c}\text { Apples } \\
\text { Concord Grapes }^{(\mathrm{f})} \\
\text { Cherries }\end{array}$ & $\begin{array}{l}\text { TE (2006) } \\
\text { TE (2007) } \\
\text { TE (2008) }\end{array}$ & $\begin{array}{l}\text { September } \\
\text { September } \\
\text { June }\end{array}$ & $\begin{array}{l}{ }^{90} \mathrm{Sr}, \text { Gamma Scan, } \mathrm{DOH}^{(\mathrm{d})} \\
{ }^{90} \mathrm{Sr} \text {, Gamma Scan } \\
{ }^{90} \mathrm{Sr} \text {, Gamma Scan }\end{array}$ \\
\hline Riverview Area & $\begin{array}{l}\text { Apples } \\
\text { Concord Grapes }^{(\mathrm{f})} \\
\text { Cherries }^{\text {Cherien }}\end{array}$ & $\begin{array}{l}\text { TE (2006) } \\
\text { TE (2007) } \\
\text { TE (2008) }\end{array}$ & $\begin{array}{l}\text { September } \\
\text { September } \\
\text { June }\end{array}$ & $\begin{array}{l}{ }^{90} \mathrm{Sr}, \text { Gamma Scan, } \mathrm{DOH},{ }^{(\mathrm{d})} \mathrm{FDA}^{(\mathrm{e})} \\
{ }^{90} \mathrm{Sr}, \text { Gamma Scan, DOH },{ }^{(\mathrm{d})} \mathrm{FDA}^{(\mathrm{e})} \\
{ }^{90} \mathrm{Sr} \text {, Gamma Scan }\end{array}$ \\
\hline Mattawa Area & Apples & TE (2006) & September & ${ }^{90} \mathrm{Sr}$, Gamma Scan, DOH ${ }^{(\mathrm{d})}$ \\
\hline Cold Creek Area & Concord Grapes $^{(\mathrm{f})}$ & TE (2007) & September & ${ }^{90} \mathrm{Sr}$, Gamma Scan \\
\hline Ringold Area & Cherries & TE (2008) & June & ${ }^{90} \mathrm{Sr}$, Gamma Scan, $\mathrm{DOH}^{(\mathrm{d})}$ \\
\hline East Wahluke Area & Cherries & TE (2008) & June & ${ }^{90} \mathrm{Sr}$, Gamma Scan \\
\hline $\begin{array}{l}\text { (a) Refer to Figure } \\
\text { (b) Two samples co } \\
\text { (c) Samples are col } \\
\text { (d) One co-sample } \\
\text { (e) Two co-samples } \\
\text { (f) Concord grapes }\end{array}$ & $\begin{array}{l}006 \text { Food and Farm } \\
\text { d for PNNL within } \\
\text { in } 2006 \text { according } t \\
\text { led to the Washingto } \\
\text { to U.S. Food and Dr } \\
\text { rred; table grapes ac }\end{array}$ & $\begin{array}{l}\text { ct Sampling Lc } \\
\text { ea, one sample } \\
\text { specified freq } \\
\text { e Department } \\
\text { ministration. } \\
\text { le if concord g }\end{array}$ & $\begin{array}{l}\text { ns. } \\
\text { yzed and one } \\
\text { unless othe } \\
\text { alth. } \\
\text { are unavaila }\end{array}$ & $\begin{array}{l}\text { ived. } \\
\text { noted. }\end{array}$ \\
\hline
\end{tabular}

\subsubsection{Wine}

\begin{tabular}{|c|c|c|c|c|}
\hline Location $^{(\mathrm{a})(\mathrm{b})}$ & $\underline{\text { Sample Type }}$ & Frequency & $\begin{array}{c}\text { Collection } \\
\text { Period }\end{array}$ & Analyses/Co-Sample \\
\hline \multirow[t]{2}{*}{ Columbia Basin } & White & A & December & Lo ${ }^{3} \mathrm{H}$, Gamma Scan, $\mathrm{DOH}^{(\mathrm{c})}$ \\
\hline & Red & A & December & Lo ${ }^{3} \mathrm{H}$, Gamma Scan, $\mathrm{DOH}^{(\mathrm{c})}$ \\
\hline \multirow[t]{2}{*}{ Yakima Valley } & White & A & December & Lo ${ }^{3} \mathrm{H}$, Gamma Scan, $\mathrm{DOH}^{(\mathrm{c})}$ \\
\hline & Red & A & December & Lo ${ }^{3} \mathrm{H}$, Gamma Scan, $\mathrm{DOH}^{(\mathrm{c})}$ \\
\hline
\end{tabular}

(a) Refer to Figure 3.1, 2006 Food and Farm Product Sampling Locations.

(b) Two samples of each type collected for PNNL within each area.

(c) One co-sample provided to the Washington State Department of Health. 


\subsubsection{Alfalfa}

\begin{tabular}{|c|c|c|c|c|}
\hline Location $^{(a)}$ & $\underline{\text { Sample Type }}$ & Frequency & $\begin{array}{c}\text { Collection } \\
\text { Period } \\
\end{array}$ & Analyses/Co-Sample \\
\hline Sagemoor Area & Alfalfa & $\mathrm{BE}(2007)$ & May & \multirow{4}{*}{$\begin{array}{l}{ }^{90} \mathrm{Sr}, \text { Gamma Scan, } \mathrm{DOH}^{(\mathrm{b})} \\
{ }^{90} \mathrm{Sr}, \text { Gamma Scan, } \mathrm{DOH}^{(\mathrm{b})} \mathrm{FDA}^{(\mathrm{c})} \\
{ }^{90} \mathrm{Sr}, \text { Gamma Scan, FDA }{ }^{(\mathrm{c})} \\
{ }^{90} \mathrm{Sr}, \text { Gamma Scan, } \mathrm{DOH}^{(\mathrm{b})}\end{array}$} \\
\hline Riverview Area & Alfalfa & BE (2007) & May & \\
\hline Sunnyside Area & Alfalfa & BE (2007) & May & \\
\hline Horn Rapids Area & Alfalfa & BE (2007) & May & \\
\hline
\end{tabular}

(a) Two samples collected for PNNL within each area, one sample analyzed and one archived.

(b) One co-sample provided to the Washington State Department of Health

(c) Two co-samples sent to U.S. Food and Drug Administration.

\subsubsection{Honey}

\begin{tabular}{|c|c|c|c|c|}
\hline Location $^{(a)}$ & Sample Type & Frequency & $\begin{array}{c}\text { Collection } \\
\text { Period } \\
\end{array}$ & Analyses/Co-Sample \\
\hline East Wahluke Area & Honey & BE (2007) & Aug-Oct & ${ }^{90} \mathrm{Sr}, \mathrm{Pu}, \mathrm{U}, \mathrm{Gamma}$ Scan, DOH ${ }^{(\mathrm{b})}$ \\
\hline Yakima Valley & Honey & $\mathrm{BE}(2007)$ & Aug-Oct & ${ }^{90} \mathrm{Sr}, \mathrm{Pu}, \mathrm{U}, \mathrm{Gamma}$ Scan, $\mathrm{DOH}^{(\mathrm{b})}$ \\
\hline
\end{tabular}

(a) One sample collected for PNNL within each area.

(b) One co-sample provided to the Washington State Department of Health. 


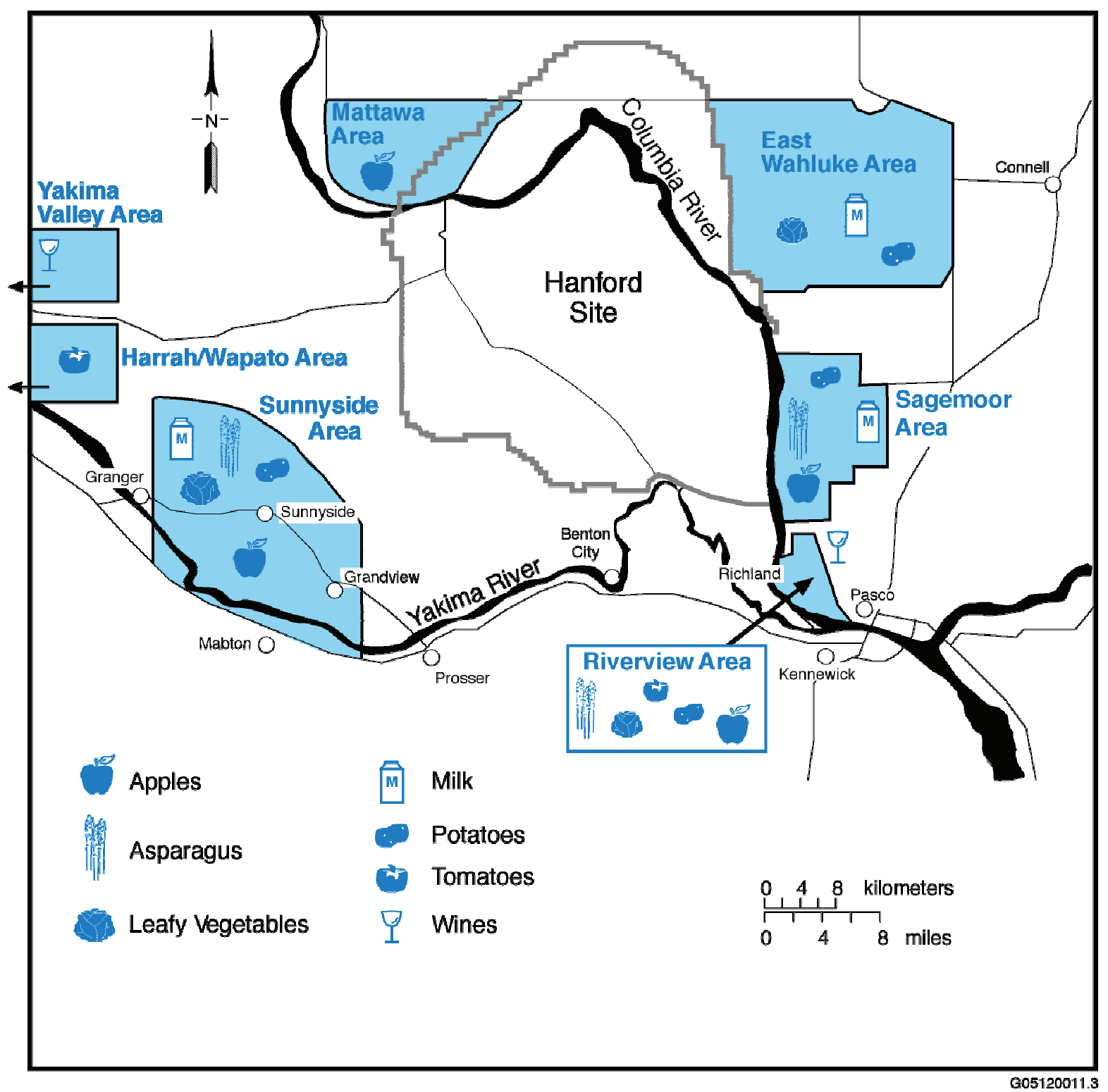

Figure 3.1. 2006 Food and Farm Product Sampling Locations 


\subsection{Wildlife}

\subsubsection{Aquatic Biota}

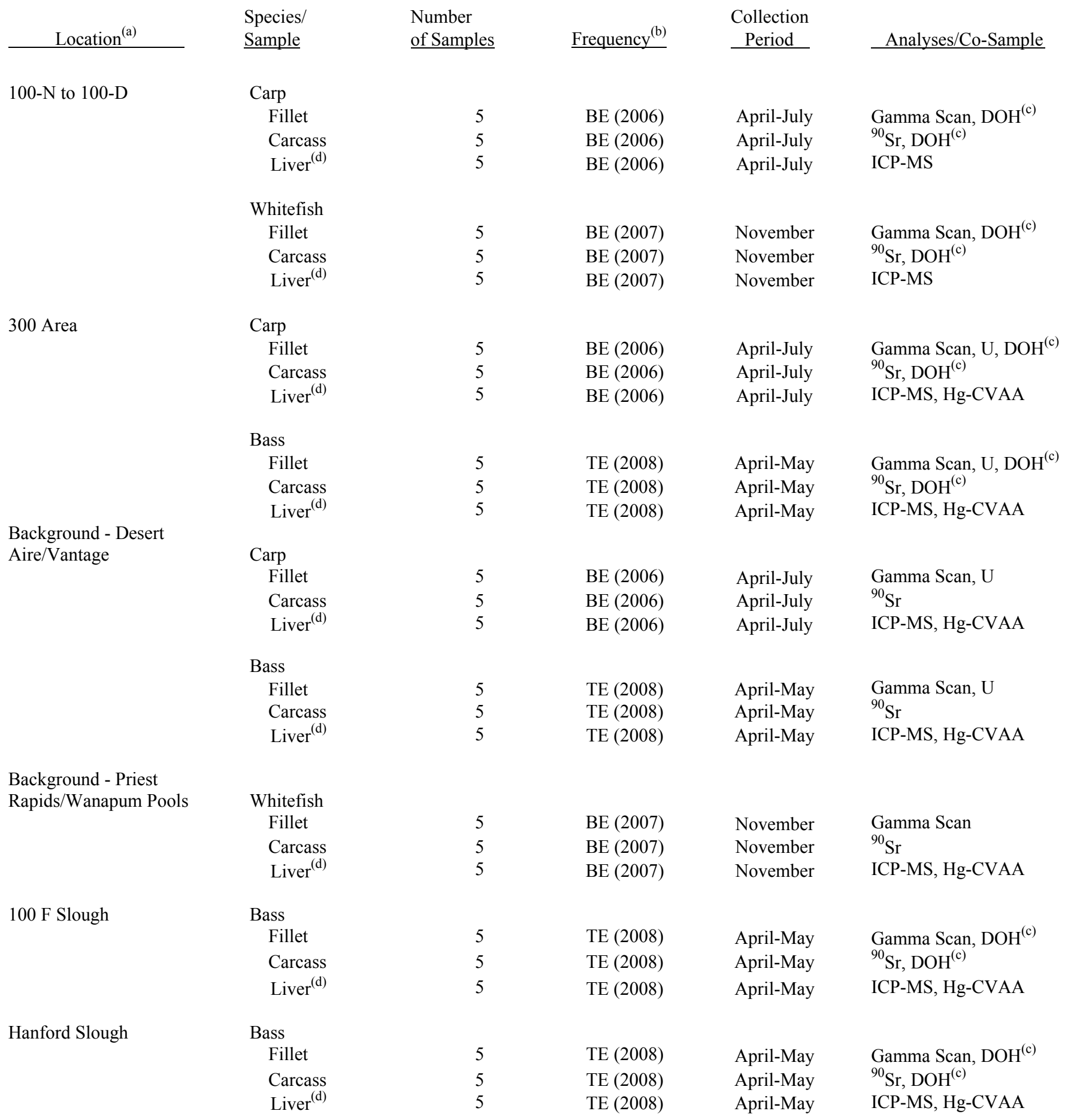

(a) Refer to Figure 3.2, 2006 Wildlife Sampling Locations.

(b) Samples are collected in 2006 according to their specified frequency unless otherwise noted.

(c) One co-sample provided to the Washington State Department of Health.

(d) Ecological assessment sample. 


\subsubsection{Geese}

\begin{tabular}{|c|c|c|c|c|c|}
\hline Location & $\underline{\text { Species/Sample }}$ & $\begin{array}{c}\begin{array}{c}\text { Number } \\
\text { of Samples }\end{array} \\
\end{array}$ & $\underline{\text { Frequency }}$ & $\begin{array}{l}\text { Collection } \\
\text { Period } \\
\end{array}$ & Analyses \\
\hline \multirow[t]{5}{*}{100 Areas } & Canada Goose & & & & \multirow{4}{*}{$\begin{array}{l}\text { Gamma Scan } \\
{ }^{90} \mathrm{Sr} \\
\mathrm{ICP}-\mathrm{MS}, \mathrm{Hg}-\mathrm{CVAA}\end{array}$} \\
\hline & Muscle & 5 & BE (2007) & May-July & \\
\hline & Bone & 5 & BE (2007) & May-July & \\
\hline & Liver $^{(a)}$ & 5 & BE (2007) & May-July & \\
\hline & Eggshells $^{(a)}$ & 10 & BE (2007) & May-June & ${ }^{90} \mathrm{Sr}$ \\
\hline \multirow[t]{5}{*}{$\begin{array}{l}\text { Hanf Townsite to } \\
300 \text { Area }\end{array}$} & Canada Goose & & & & \multirow{4}{*}{$\begin{array}{l}\text { Gamma Scan } \\
{ }^{90} \mathrm{Sr} \\
\text { ICP-MS, Hg-CVAA }\end{array}$} \\
\hline & Muscle & 5 & BE (2007) & May-July & \\
\hline & Bone & 5 & BE (2007) & May-July & \\
\hline & Liver $^{(a)}$ & 5 & BE (2007) & May-July & \\
\hline & Eggshells $^{(a)}$ & 10 & BE (2007) & May-June & ${ }^{90} \mathrm{Sr}$ \\
\hline \multirow[t]{5}{*}{ Background -Vantage } & Canada Goose & & & & \multirow{4}{*}{$\begin{array}{l}\text { Gamma Scan } \\
{ }^{90} \mathrm{Sr} \\
\text { ICP-MS, Hg-CVAA }\end{array}$} \\
\hline & Muscle & 5 & BE (2007) & May-July & \\
\hline & Bone & 5 & BE (2007) & May-July & \\
\hline & Liver $^{(a)}$ & 5 & BE (2007) & May-July & \\
\hline & Eggshells $^{(a)}$ & 10 & BE (2007) & May-June & ${ }^{90} \mathrm{Sr}$ \\
\hline
\end{tabular}

(a) Ecological assessment sample.

\subsubsection{Game Birds}

\begin{tabular}{|c|c|c|c|c|c|}
\hline Location $^{(a)}$ & $\underline{\text { Species/Sample }}{ }^{(\mathrm{b})}$ & $\begin{array}{c}\begin{array}{c}\text { Number } \\
\text { of Samples }\end{array} \\
\end{array}$ & $\underline{\text { Frequency }}$ & $\begin{array}{c}\text { Collection } \\
\text { Period }\end{array}$ & Analyses/Co-Sample \\
\hline \multirow[t]{4}{*}{$100-\mathrm{D}$ to $100-\mathrm{H}$} & Pheasant & & & & \multirow{4}{*}{$\begin{array}{l}\text { Gamma Scan } \\
{ }^{90} \mathrm{Sr} \\
\text { ICP-MS }\end{array}$} \\
\hline & Muscle & 4 & BE (2006) & September & \\
\hline & Bone & 4 & BE (2006) & September & \\
\hline & Liver $^{(c)}$ & 4 & $\mathrm{BE}(2006)$ & September & \\
\hline \multirow[t]{4}{*}{$100-\mathrm{H}$ to $100-\mathrm{F}$} & Pheasant & & & & \multirow{4}{*}{$\begin{array}{l}\text { Gamma Scan, } \mathrm{DOH}^{(\mathrm{d})} \\
{ }^{90} \mathrm{Sr}, \mathrm{DOH}^{(\mathrm{d})} \\
\text { ICP-MS }\end{array}$} \\
\hline & Muscle & 6 & BE (2006) & September & \\
\hline & Bone & 6 & BE (2006) & September & \\
\hline & Liver $^{(\mathrm{c})}$ & 6 & BE (2006) & September & \\
\hline \multirow[t]{4}{*}{ Background } & Pheasant & & & & \multirow{4}{*}{$\begin{array}{l}\text { Gamma Scan, } \mathrm{DOH}^{(\mathrm{d})} \\
{ }^{90} \mathrm{Sr}, \mathrm{DOH}^{(\mathrm{d})} \\
\text { ICP-MS }\end{array}$} \\
\hline & Muscle & 5 & BE (2006) & September & \\
\hline & Bone & 5 & BE (2006) & September & \\
\hline & Liver $^{(\mathrm{c})}$ & 5 & BE (2006) & September & \\
\hline
\end{tabular}

(a) Refer to Figure 3.2, 2006 Wildlife Sampling Locations.

(b) Pheasant preferred; chukars or quail acceptable if pheasants are unavailable.

(c) Ecological assessment sample.

(d) One co-sample provided to the Washington State Department of Health. 


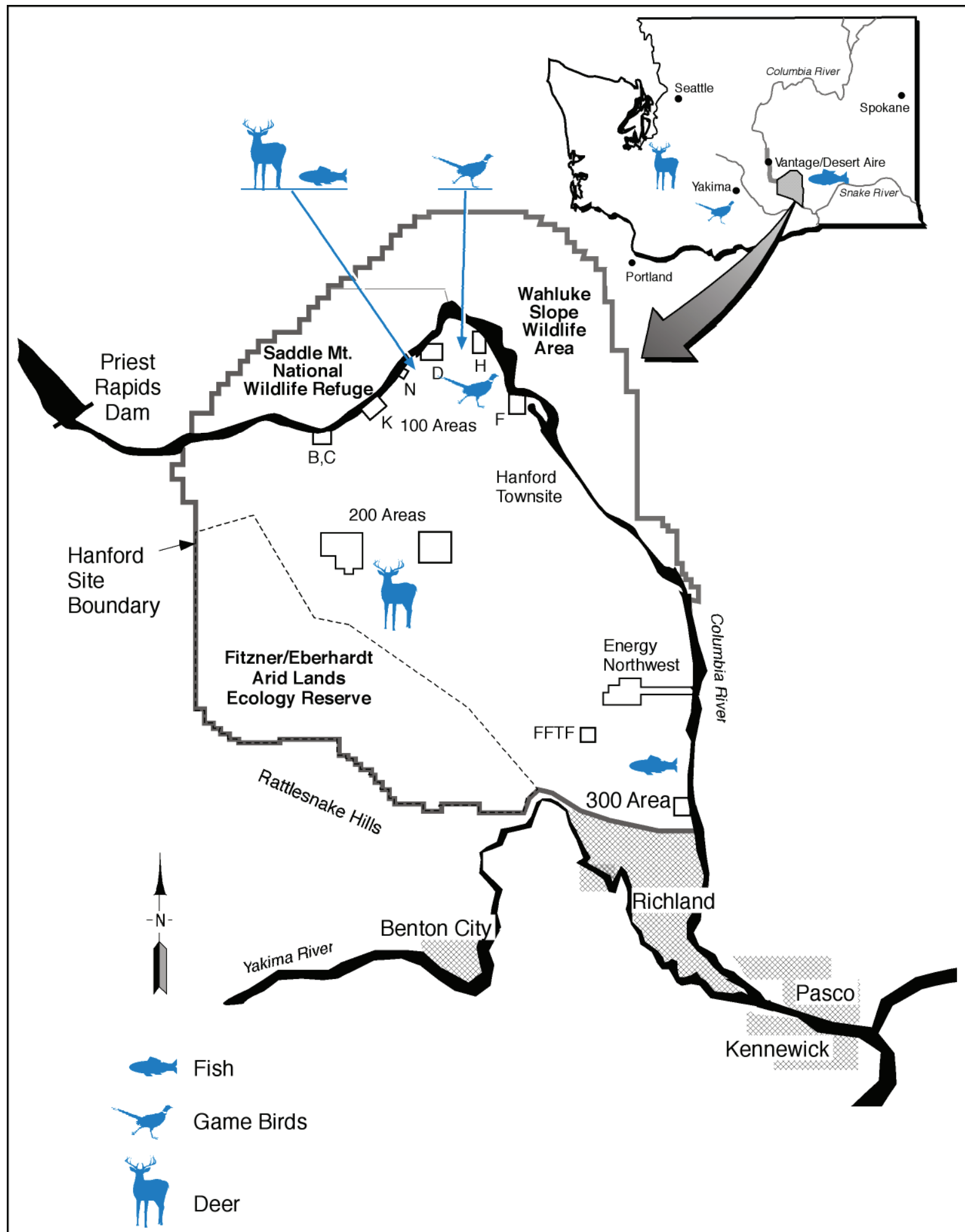

Not to Scale

G05120011.4

Figure 3.2. 2006 Wildlife Sampling Locations 


\subsection{Soil and Vegetation}

4.1 Soil

\begin{tabular}{|c|c|c|c|}
\hline Location & Frequency $^{(a)}$ & $\begin{array}{l}\text { Collection } \\
\text { Period }\end{array}$ & Analyses/Co-Sample \\
\hline $100 \mathrm{~K}$ Area & 3 to $5 \mathrm{yrs}$ & June-Sept & Gamma Scan, ${ }^{90} \mathrm{Sr}, \mathrm{U}, \mathrm{Pu}, \mathrm{DOH}^{(\mathrm{b})}$ \\
\hline NE of $100 \mathrm{~N}$ Area & 3 to 5 yrs & June-Sept & Gamma Scan, ${ }^{90} \mathrm{Sr}, \mathrm{U}, \mathrm{Pu}$ \\
\hline E of $100 \mathrm{~N}$ Area & 3 to $5 \mathrm{yrs}$ & June-Sept & Gamma Scan, ${ }^{90} \mathrm{Sr}, \mathrm{U}, \mathrm{Pu}, \mathrm{DOH}^{(\mathrm{b})}$ \\
\hline 100N Shore Above HGP & 3 to $5 \mathrm{yrs}$ & June-Sept & Gamma Scan, ${ }^{90} \mathrm{Sr}, \mathrm{U}, \mathrm{Pu}$ \\
\hline $100 \mathrm{~N}$ Spring Shoreline & 3 to $5 \mathrm{yrs}$ & June-Sept & Gamma Scan, ${ }^{90} \mathrm{Sr}, \mathrm{U}, \mathrm{Pu}$ \\
\hline Above 100D Pumphouse & 3 to $5 \mathrm{yrs}$ & June-Sept & Gamma Scan, ${ }^{90} \mathrm{Sr}, \mathrm{U}, \mathrm{Pu}$ \\
\hline 100 Area Fire Stat & 3 to $5 \mathrm{yrs}$ & June-Sept & Gamma Scan, ${ }^{90} \mathrm{Sr}, \mathrm{U}, \mathrm{Pu}$ \\
\hline $200 \mathrm{ENC}$ & 3 to $5 \mathrm{yrs}$ & June-Sept & Gamma Scan, ${ }^{90} \mathrm{Sr}, \mathrm{U}, \mathrm{Pu}$ \\
\hline E of $200 \mathrm{E}$ & 3 to $5 \mathrm{yrs}$ & June-Sept & Gamma Scan, ${ }^{90} \mathrm{Sr}, \mathrm{U}, \mathrm{Pu}, \mathrm{DOH}(\mathrm{b})$ \\
\hline $200 \mathrm{ESE}$ & 3 to 5 yrs & June-Sept & Gamma Scan, ${ }^{90} \mathrm{Sr}, \mathrm{U}, \mathrm{Pu},{ }^{241} \mathrm{Am}$ \\
\hline$S$ of $200 \mathrm{E}$ & 3 to $5 \mathrm{yrs}$ & June-Sept & Gamma Scan, ${ }^{90} \mathrm{Sr}, \mathrm{U}, \mathrm{Pu}$ \\
\hline $\mathrm{SW}$ of $\mathrm{B} / \mathrm{C}$ Cribs & 3 to $5 \mathrm{yrs}$ & June-Sept & Gamma Scan, ${ }^{90} \mathrm{Sr}, \mathrm{U}, \mathrm{Pu},{ }^{241} \mathrm{Am}, \mathrm{DOH}^{(\mathrm{b})}$ \\
\hline E of $200 \mathrm{~W}$ Gate & 3 to 5 yrs & June-Sept & Gamma Scan, ${ }^{90} \mathrm{Sr}, \mathrm{U}, \mathrm{Pu},{ }^{241} \mathrm{Am}, \mathrm{DOH}^{(\mathrm{b})}$ \\
\hline S of $200 \mathrm{~W}$ & 3 to 5 yrs & June-Sept & Gamma Scan, ${ }^{90} \mathrm{Sr}, \mathrm{U}, \mathrm{Pu}, \mathrm{DOH}^{(\mathrm{b})}$ \\
\hline Rattlesnake Springs & 3 to $5 \mathrm{yrs}$ & June-Sept & Gamma Scan, ${ }^{90} \mathrm{Sr}, \mathrm{U}, \mathrm{Pu}, \mathrm{DOH}^{(\mathrm{b})}$ \\
\hline Yakima Barricade & 3 to $5 \mathrm{yrs}$ & June-Sept & Gamma Scan, ${ }^{90} \mathrm{Sr}, \mathrm{U}, \mathrm{Pu}$ \\
\hline $400 \mathrm{E}$ & 3 to 5 yrs & June-Sept & Gamma Scan, ${ }^{90} \mathrm{Sr}, \mathrm{U}, \mathrm{Pu}$ \\
\hline SE Side of FFTF & 3 to 5 yrs & June-Sept & Gamma Scan, ${ }^{90} \mathrm{Sr}, \mathrm{U}, \mathrm{Pu}, \mathrm{DOH}^{(\mathrm{b})}$ \\
\hline North of 300 Area & 3 to $5 \mathrm{yrs}$ & June-Sept & Gamma Scan, ${ }^{90} \mathrm{Sr}, \mathrm{U}, \mathrm{Pu}, \mathrm{DOH}^{(\mathrm{b})}$ \\
\hline South of 300 Area & 3 to $5 \mathrm{yrs}$ & June-Sept & Gamma Scan, ${ }^{90} \mathrm{Sr}, \mathrm{U}, \mathrm{Pu}, \mathrm{DOH}^{(\mathrm{b})}$ \\
\hline Hanford Townsite & 3 to 5 yrs & June-Sept & Gamma Scan, ${ }^{90} \mathrm{Sr}, \mathrm{U}, \mathrm{Pu}$ \\
\hline Wye Barricade ${ }^{(c)}$ & 3 to $5 \mathrm{yrs}$ & June-Sept & Gamma Scan, ${ }^{90} \mathrm{Sr}, \mathrm{U}, \mathrm{Pu}$ \\
\hline Prosser Barricade & 3 to $5 \mathrm{yrs}$ & June-Sept & Gamma Scan, ${ }^{90} \mathrm{Sr}, \mathrm{U}, \mathrm{Pu}$ \\
\hline ALE Field Lab & 3 to $5 \mathrm{yrs}$ & June-Sept & Gamma Scan, ${ }^{90} \mathrm{Sr}, \mathrm{U}, \mathrm{Pu}$ \\
\hline N End Vernita Bridge & 3 to 5 yrs & June-Sept & Gamma Scan, ${ }^{90} \mathrm{Sr}, \mathrm{U}, \mathrm{Pu}$ \\
\hline Wahluke Slope & 3 to $5 \mathrm{yrs}$ & June-Sept & Gamma Scan, ${ }^{90} \mathrm{Sr}, \mathrm{U}, \mathrm{Pu}$ \\
\hline Berg Ranch & 3 to $5 \mathrm{yrs}$ & June-Sept & Gamma Scan, ${ }^{90} \mathrm{Sr}, \mathrm{U}, \mathrm{Pu}$ \\
\hline Ringold Area & 3 to $5 \mathrm{yrs}$ & June-Sept & Gamma Scan, ${ }^{90} \mathrm{Sr}, \mathrm{U}, \mathrm{Pu}$ \\
\hline W End of Fir Road & 3 to $5 \mathrm{yrs}$ & June-Sept & Gamma Scan, ${ }^{90} \mathrm{Sr}, \mathrm{U}, \mathrm{Pu}$ \\
\hline Taylor Flats No. 2 & 3 to 5 yrs & June-Sept & Gamma Scan, ${ }^{90} \mathrm{Sr}, \mathrm{U}, \mathrm{Pu}$ \\
\hline Sagemoor Farm ${ }^{(\mathrm{c})}$ & 3 to 5 yrs & June-Sept & Gamma Scan, ${ }^{90} \mathrm{Sr}, \mathrm{U}, \mathrm{Pu},{ }^{241} \mathrm{Am}$ \\
\hline Byers Landing & 3 to $5 \mathrm{yrs}$ & June-Sept & Gamma Scan, ${ }^{90} \mathrm{Sr}, \mathrm{U}, \mathrm{Pu}$ \\
\hline Riverview-Harris & 3 to $5 \mathrm{yrs}$ & June-Sept & Gamma Scan, ${ }^{90} \mathrm{Sr}, \mathrm{U}, \mathrm{Pu}$ \\
\hline Benton City & 3 to $5 \mathrm{yrs}$ & June-Sept & Gamma Scan, ${ }^{90} \mathrm{Sr}, \mathrm{U}, \mathrm{Pu}$ \\
\hline Sunnyside & 3 to $5 \mathrm{yrs}$ & June-Sept & Gamma Scan, ${ }^{90} \mathrm{Sr}, \mathrm{U}, \mathrm{Pu},{ }^{241} \mathrm{Am}$ \\
\hline McNary Dam & 3 to $5 \mathrm{yrs}$ & June-Sept & Gamma Scan, ${ }^{90} \mathrm{Sr}, \mathrm{U}, \mathrm{Pu}$ \\
\hline Walla Walla & 3 to $5 \mathrm{yrs}$ & June-Sept & Gamma Scan, ${ }^{90} \mathrm{Sr}, \mathrm{U}, \mathrm{Pu}$ \\
\hline Washtucna & 3 to $5 \mathrm{yrs}$ & June-Sept & Gamma Scan, ${ }^{90} \mathrm{Sr}, \mathrm{U}, \mathrm{Pu}$ \\
\hline Toppenish & 3 to $5 \mathrm{yrs}$ & June-Sept & Gamma Scan, ${ }^{90} \mathrm{Sr}, \mathrm{U}, \mathrm{Pu}$ \\
\hline George & 3 to $5 \mathrm{yrs}$ & June-Sept & Gamma Scan, ${ }^{90} \mathrm{Sr}, \mathrm{U}, \mathrm{Pu}, \mathrm{DOH}^{(\mathrm{b})}$ \\
\hline Othello & 3 to 5 yrs & June-Sept & Gamma Scan, ${ }^{90} \mathrm{Sr}, \mathrm{U}, \mathrm{Pu}, \mathrm{DOH}^{(\mathrm{b})}$ \\
\hline Wanapum & 3 to $5 \mathrm{yrs}$ & June-Sept & Gamma Scan, ${ }^{90} \mathrm{Sr}, \mathrm{U}, \mathrm{Pu}, \mathrm{DOH}^{(\mathrm{b})}$ \\
\hline
\end{tabular}

(a) Samples are collected once every 3 to 5 years and will be collected in 2007 .

(b) One co-sample provided to the Washington State Department of Health.

(c) Quality assurance samples submitted for analyses. 


\subsection{Vegetation}

\begin{tabular}{|c|c|c|c|}
\hline Location & Frequency $^{(\mathrm{a})}$ & $\begin{array}{c}\text { Collection } \\
\text { Period } \\
\end{array}$ & Analyses/Co-Sample \\
\hline $100 \mathrm{~K}$ Area & 3 to $5 \mathrm{yrs}$ & June-Sept & Gamma Scan, ${ }^{90} \mathrm{Sr}, \mathrm{U}, \mathrm{Pu}, \mathrm{DOH}^{(\mathrm{b})}$ \\
\hline NE of $100 \mathrm{~N}$ Area & 3 to $5 \mathrm{yrs}$ & June-Sept & Gamma Scan, ${ }^{90} \mathrm{Sr}, \mathrm{U}, \mathrm{Pu}, \mathrm{DOH}^{(\mathrm{b})}$ \\
\hline E of $100 \mathrm{~N}$ Area & 3 to $5 \mathrm{yrs}$ & June-Sept & Gamma Scan, ${ }^{90} \mathrm{Sr}, \mathrm{U}, \mathrm{Pu}$ \\
\hline $100 \mathrm{~N}$ Spring Shoreline & 3 to $5 \mathrm{yrs}$ & June-Sept & Gamma Scan, ${ }^{90} \mathrm{Sr}, \mathrm{U}, \mathrm{Pu}$ \\
\hline E of $200 \mathrm{~W}$ Gate & 3 to $5 \mathrm{yrs}$ & June-Sept & Gamma Scan, ${ }^{90} \mathrm{Sr}, \mathrm{U}, \mathrm{Pu}$ \\
\hline 300 Area Shoreline & 3 to $5 \mathrm{yrs}$ & June-Sept & Gamma Scan, ${ }^{90} \mathrm{Sr}, \mathrm{U}, \mathrm{Pu}, \mathrm{DOH}^{(\mathrm{b})}$ \\
\hline Hanford Townsite & 3 to $5 \mathrm{yrs}$ & June-Sept & Gamma Scan, ${ }^{90} \mathrm{Sr}, \mathrm{U}, \mathrm{Pu}$ \\
\hline Hanford Twnsite HRM28 & 3 to $5 \mathrm{yrs}$ & June-Sept & Gamma Scan, ${ }^{90} \mathrm{Sr}, \mathrm{U}, \mathrm{Pu}, \mathrm{DOH}^{(\mathrm{b})}$ \\
\hline Ringold Area & 3 to $5 \mathrm{yrs}$ & June-Sept & Gamma Scan, ${ }^{90} \mathrm{Sr}, \mathrm{U}, \mathrm{Pu}$ \\
\hline Sagemoor Farm & 3 to $5 \mathrm{yrs}$ & June-Sept & Gamma Scan, ${ }^{90} \mathrm{Sr}, \mathrm{U}, \mathrm{Pu}$ \\
\hline Byers Landing & 3 to $5 \mathrm{yrs}$ & June-Sept & Gamma Scan, ${ }^{90} \mathrm{Sr}, \mathrm{U}, \mathrm{Pu}$ \\
\hline Riverview-Harris & 3 to 5 yrs & June-Sept & Gamma Scan, ${ }^{90} \mathrm{Sr}, \mathrm{U}, \mathrm{Pu}$ \\
\hline Sunnyside & 3 to 5 yrs & June-Sept & Gamma Scan, ${ }^{90} \mathrm{Sr}, \mathrm{U}, \mathrm{Pu}$ \\
\hline Toppenish & 3 to 5 yrs & June-Sept & Gamma Scan, ${ }^{90} \mathrm{Sr}, \mathrm{U}, \mathrm{Pu}$ \\
\hline George & 3 to $5 \mathrm{yrs}$ & June-Sept & Gamma Scan, ${ }^{90} \mathrm{Sr}, \mathrm{U}, \mathrm{Pu}, \mathrm{DOH}^{(\mathrm{b})}$ \\
\hline Othello & 3 to $5 \mathrm{yrs}$ & June-Sept & Gamma Scan, ${ }^{90} \mathrm{Sr}, \mathrm{U}, \mathrm{Pu}, \mathrm{DOH}^{(\mathrm{b})}$ \\
\hline Wanapum & 3 to $5 \mathrm{yrs}$ & June-Sept & Gamma Scan, ${ }^{90} \mathrm{Sr}, \mathrm{U}, \mathrm{Pu}, \mathrm{DOH}^{(\mathrm{b})}$ \\
\hline
\end{tabular}

(a) Samples are collected once every 3 to 5 years and will be collected in 2007 .

(b) One co-sample provided to the Washington State Department of Health. 


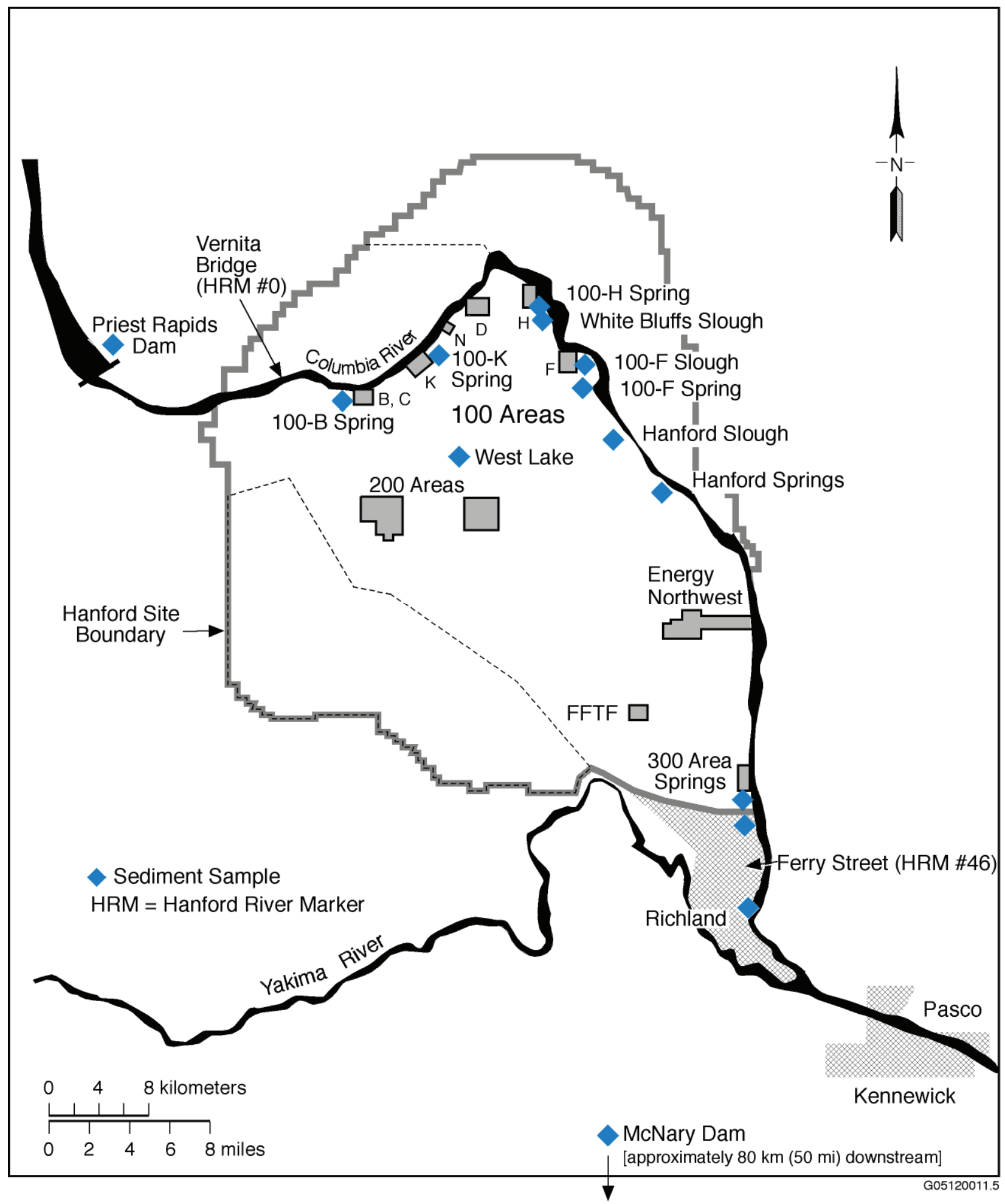

Figure 5.1. 2006 Sediment Sampling Locations 


\subsection{References}

DOE. 1991. Environmental Monitoring Plan, United States Department of Energy, U.S. Department of Energy, Richland Operations Office. DOE/RL-91-50, U.S. Department of Energy, Richland, Washington.

DOE Order 450.1. Environmental Protection Program.

DOE Order 5400.5. Radiation Protection of the Public and the Environment. 


\section{Distribution}

No. of

Copies

OFFSITE (23)

2 L. Albin

Division of Radiation Protection

Washington State Dept. of Health

P.O. Box 47827

Olympia, WA 98504-7827

R. Buck, Jr.

Wanapum

P.O. Box 878

Ephrata, WA 98823

2 N. Ceto

U.S. Environmental Protection Agency,

Hanford Project Office

309 Bradley, Suite 115, MS B1-46

Richland, WA 99352

S. Harris

Confederated Tribes of the Umatilla Indian

Reservation

P.O. Box 638

Pendleton, OR 97801

G. Hughes

U.S. Fish and Wildlife Service

3250 Port of Benton Boulevard

Richland, WA 99354-1670

R. Jim, Manager

Environmental Restoration and Waste

Management Program

The Confederated Tribes and Bands of the

Yakama Nation

P.O. Box 151

Toppenish, WA 98948

D. Landeen

Hanford Natural Resources Trustee Council

Nez Perce Tribe

P.O. Box 365

Lapwai, ID 83540
No. of

$\underline{\text { Copies }}$

S. Langford

Division of Radiation Protection

Washington State Dept. of Health

P.O. Box 47827

Olympia, WA 98504-7827

D. McBaugh

Division of Radiation Protection

Washington State Department of Health

P.O. Box 47827

Olympia, WA 98504-7827

Office of Environmental Cleanup

Director - Hanford Project Office

$12006^{\text {th }}$ Avenue

Seattle, WA 98101

C. Palmer

The Confederated Tribes and Bands of the Yakama Nation

Department of Natural Resources

P.O. Box 151

Toppenish, WA 98948

C. Pleasants

Confederated Tribes of the Colville Reservation

P.O. Box 150

Nespelem, WA 99155

M. Priddy

Washington State Department of Health

309 Bradley, Suite 201

Richland, WA 99352

M. Ritter

U.S. Fish and Wildlife Service

3250 Port of Benton Boulevard

Richland, WA 99354

B. Ruben

Washington State Department of Health

309 Bradley, Suite 201

Richland, WA 99352

Distr. 1 
PNNL-15618

No. of

Copies

L. Seelatsee

Wanapum

P.O. Box 878

Ephrata, WA 98823

P. Sobotta

Environmental Restoration and Waste

Management Program

Nez Perce Tribe

P.O. Box 365

Lapwai, ID 83540

T. Southworth

Energy Northwest

P.O. Box 968

Richland, WA 99354

E. Stensgar

Coeur d'Alene Tribal Council

P.O. Box 408

Plummer, ID 83851-9704

U.S. Environmental Protection Agency, Hanford Project Office

309 Bradley, Suite 115 MS B1-46

Richland WA 99352

S.P. Van Verst

Division of Radiation Protection

Washington State Department of Health

P.O. Box 47827

Olympia, WA 98504-7827

\section{ONSITE (97)}

\section{DOE Richland Operations}

L. Erickson

A3-04

K.A. Klein

A7 -50

J.P. Sands

A3-04

K.M. Thompson

A6-38

A.C. Tortoso

A6-38

D.C. Ward (5)

A2-17

S.H. Wisness

A3-04

J. Zeisloft

A3-04

DOE Public Reading Room (2)

$\mathrm{H} 2-53$

DOE Office of River Protection

W. Russell

H6-60
No. of

Copies

Duratek Federal Services of Hanford

L.P. Diediker

H8-13

$4 \quad$ Duratek Federal Services Northwest

J.J. Dorian

H1-11

S.M. McKinney

H1-11

R.M. Mitchell

H1-11

C.J. Perkins

H1-11

$3 \quad$ Fluor Hanford

D.L. Dyekman

H8-13

A.R. Johnson

H5-26

L.M. Kelly

S4-21

3 Washington Closure Hanford

K.A. Gano

H9-03

R.W. Ovink

H9-01

J.E. Thomson

$\mathrm{H} 0-23$

71 Pacific Northwest National Laboratory

E.J. Antonio

K3-54

L.E. Bisping (30)

K6-75

J.M. Brandenburger

Sequim

A.T. Cooper

K6-75

R.L. Dirkes (20)

K6-75

J.L. Downs

K6-85

P.E. Dresel

K6-96

B.G. Fritz

K6-75

R.W. Fulton

K6-75

R.W. Hanf, Jr.

K6-75

M.J. Hartman

K6-96

B.K Lasora

E.A. Lepel

Sequim

P8-01

B.E. Opitz

K6-75

G.W. Patton

K6-75

R.E. Peterson

K6-75

T.M. Poston

K6-75

E.L. Radford

K6-75

J.A. Stegen

K6-85

R.K. Zufelt

K6-85

Historical File-T.M. Poston K6-75

Hanford Technical Library (2) P8-55

Distr. 2 